\title{
An Improved-time Polynomial-space Exact Algorithm for TSP in Degree-5 Graphs
}

\author{
Norhazwani Md Yunos ${ }^{1, a)}$ Aleksandar Shurbevski ${ }^{2, b)}$ Hiroshi Nagamochi ${ }^{2, c)}$ \\ Received: November 7, 2016, Accepted: May 16, 2017
}

\begin{abstract}
The Traveling Salesman Problem (TSP) is one of the most well-known NP-hard optimization problems. Following a recent trend of research which focuses on developing algorithms for special types of TSP instances, namely graphs of limited degree, in an attempt to reduce a part of the time and space complexity, we present a polynomialspace branching algorithm for the TSP in an $n$-vertex graph with degree at most 5 , and show that it has a running time of $O^{*}\left(2.3500^{n}\right)$, which improves the previous best known time bound of $O^{*}\left(2.4723^{n}\right)$ given by the authors (the $12^{\text {th }}$ International Symposium on Operations Research and Its Application (ISORA 2015), pp.45-58, 2015). While the base of the exponent in the running time bound of our algorithm is greater than 2, it still outperforms Gurevich and Shelah's $O^{*}\left(4^{n} n^{\log n}\right)$ polynomial-space exact algorithm for the TSP in general graphs (SIAM Journal of Computation, Vol.16, No.3, pp.486-502, 1987). In the analysis of the running time, we use the measure-and-conquer method, and we develop a set of branching rules which foster the analysis of the running time.
\end{abstract}

Keywords: Traveling Salesman Problem, exact exponential algorithm, branch-and-reduce, measure-and-conquer

\section{Introduction}

The Traveling Salesman Problem (TSP) is one of the most extensively studied problems across many fields of optimization. Briefly, given a set of cities and their pairwise distances, the TSP asks for a shortest closed route which visits each of the cities exactly once. It has been formulated as a mathematical problem in the 1930s, and many algorithmic methods have been investigated to address the challenge of finding the fastest algorithm in terms of running time. On the other hand, it has proven even more challenging to devise reasonable algorithms that would use a manageable amount of computation space, bounded by a polynomial in an input instance's size. We review previous algorithmic attempts, making a distinction between those which require space exponential in the size of a problem instance, and those requiring space polynomial in the input size. We use the $O^{*}$ notation, which suppresses polynomial factors.

The first non-trivial algorithm for the TSP in an $n$-vertex graph is the $O^{*}\left(2^{n}\right)$-time dynamic programming algorithm discovered independently by Bellman [2], and Held and Karp [10] in the early 1960s. This dynamic programming algorithm however, requires also an exponential amount of space. Ever since, this running time has only been improved for special types of graphs. Primarily, investigation efforts have been focused on graphs in

\footnotetext{
Technical University of Malaysia Malacca, Malacca 76900, Malaysia

Department of Applied Mathematics and Physics, Graduate School of Informatics, Kyoto University, Sakyo, Kyoto 606-8501, Japan

a) wanie.my@utem.edu.my

b) shurbevski@amp.i.kyoto-u.ac.jp

c) nag@amp.i.kyoto-u.ac.jp

This work has been done while the first author was a Ph.D. student at the Department of Applied Mathematics and Physics, Graduate School of Informatics, Kyoto University.
}

which vertices have a limited degree. Henceforth, let degree- $i$ graph stand for a graph in which vertices have a maximum degree at most $i$. A recent improvement of the time bound to $O^{*}\left(1.2186^{n}\right)$ for degree-3 graphs has been presented by Bodlaender et al. [3], where the authors make use of a general approach for speeding up straightforward dynamic programming algorithms. For the TSP in degree-4 graphs, Gebauer [8] has shown a time bound of $O^{*}\left(1.733^{n}\right)$, by using a dynamic programming approach. The running space of these algorithms remains exponential in the size of an input instance.

In the vein of polynomial space algorithms, Gurevich and Shelah [9] have shown that the TSP in a general $n$-vertex graph is solvable in time $O^{*}\left(4^{n} n^{\log n}\right)$. Eppstein [5] started the exploration into polynomial space TSP algorithms specialized for graphs of bounded degree by designing an algorithm for degree-3 graphs that runs in $O^{*}\left(1.260^{n}\right)$ time. He introduced a branch-and-search method by considering a generalization of the TSP proposed by Rubin [14], called the forced TSP. Iwama and Nakashima [11] claimed an improvement of Eppstein's time bound to $O^{*}\left(1.251^{n}\right)$ time for the TSP in degree-3 graphs. Later, Liskiewicz and Schuster [12] uncovered some oversights made in Iwama and Nakashima's analysis, and proved that their algorithm actually runs in $O^{*}\left(1.257^{n}\right)$ time. Liskiewicz and Schuster then made some minor modifications to Eppstein's algorithm and showed that this modified algorithm runs in $O^{*}\left(1.2553^{n}\right)$ time, a slight improvement over Iwama and Nakashima's algorithm. Xiao and Nagamochi [15] have recently presented an $O^{*}\left(1.2312^{n}\right)$-time algorithm for the TSP in degree-3 graphs, and this improved all previous time bounds for polynomial-space algorithms. They used the basic steps of Eppstein's branch-and-search algorithm, and introduced a branching rule based on a cut-circuit structure. 
In the process of improving the time bound, they used a simple measure-and-conquer analysis, and effectively analyzed their algorithm by introducing an amortization scheme over the cutcircuit structure, setting weights to both vertices and connected components of induced graphs.

For the TSP in degree-4 graphs, Eppstein [5] designed an algorithm that runs in $O^{*}\left(1.890^{n}\right)$ time, based on a branch-and-search method. Following, Xiao and Nagamochi [16] showed an improved value for the upper bound of the running time and showed that their algorithm runs in $O^{*}\left(1.692^{n}\right)$ time. To the best of our knowledge, this is currently the fastest algorithm for the TSP in degree-4 graphs. Basically, the idea behind their algorithm is to apply reduction rules until no further reduction is possible, and then branch on an edge by either including it in a solution or excluding it from any solution. This is similar to most of the previous branch-and-search algorithms for the TSP. To effectively analyze their algorithm, Xiao and Nagamochi used the measureand-conquer method by setting a weight to each vertex in an input graph. From each branching operation, they derived a branching vector using the assigned weight and evaluated how much weight can be decreased in each of the two instances obtained by branching on a selected edge $e$. In this way, they were able to analyze by how much the total weight decreases in each branch. Moreover, they indicated that the measure will decrease more if we select a "good" edge to branch on, and gave a set of simple rules based on a graph's topological properties for choosing such an edge. However, the analysis of the running time itself is not as straightforward, and the interested reader is referred to the original paper [16].

Md Yunos et al. [13] presented the first algorithm specialized for the TSP in degree-5 graphs, and showed that their algorithm runs in $O^{*}\left(2.4723^{n}\right)$ time. This algorithm employs similar techniques as most of the previous branching algorithms for the TSP. In the analysis, the authors use the measure-and-conquer method as a tool to get an upper bound of the running time. In this work we present an extended algorithm coupled with an improved analysis, giving an upper bound on the running time of $O^{*}\left(2.3500^{n}\right)$ for the TSP in degree- 5 graphs with $n$ vertices, which improves the previous result by the authors [13]. Namely, a new branching rule has been introduced for convenience in the analysis, and we present a much more detailed analysis, through which we are able to obtain an improved bound on the running time.

The remainder of this paper is organized as follows. Section 2 introduces the basic notation used in this paper and presents an introduction to branching algorithms and the measure-and-conquer method. Section 3 describes our polynomial-space branching algorithm. We state our main result in Section 4, where we proceed with the analysis of the proposed algorithm. Finally, Section 5 concludes the paper.

\section{Methods}

\subsection{Preliminaries}

For a graph $G$, let $V(G)$ denote the set of vertices in $G$, and let $E(G)$ denote the set of edges in $G$. A vertex $u$ is a neighbor of a vertex $v$ if $u$ and $v$ are adjacent by an edge $u v$. We denote the set of all neighbors of a vertex $v$ by $N(v)$, also called the neighborhood of $v$, and denote by $d(v)$ the cardinality $|N(v)|$ of $N(v)$, also called the degree of $v$.

For a vertex $v$ and subsets $W \subseteq V(G)$ of vertices, and $E^{\prime} \subseteq$ $E(G)$ of edges, we consider the following sets of vertices:

- $N(v ; W) \triangleq N(v) \cap W$,

- $N_{E^{\prime}}(v) \triangleq N(v) \cap\left\{u \mid u v \in E^{\prime}\right\}$, and

- $N_{E^{\prime}}(v ; W) \triangleq N_{E^{\prime}}(v) \cap W$.

Let $d_{E^{\prime}}(v)$ and $d_{E^{\prime}}(v, W)$ denote the cardinalities $\left|N_{E^{\prime}}(v)\right|$ and $\left|N_{E^{\prime}}(v, W)\right|$ of $N_{E^{\prime}}(v)$ and $N_{E^{\prime}}(v, W)$, respectively. Also, for a subset $E^{\prime}$ of $E(G)$, we denote by $G-E^{\prime}$ the graph $\left(V, E \backslash E^{\prime}\right)$ obtained from $G$ by removing the edges in $E^{\prime}$.

We employ a known generalization of the TSP proposed by Rubin [14], named the forced Traveling Salesman Problem by Eppstein [5]. We define an instance $I=(G, F)$ that consists of a simple, edge weighted, undirected graph $G$, and a subset $F$ of edges in $G$, called forced. For brevity, throughout this paper let $U$ denote $E(G) \backslash F$. A vertex is called forced if exactly one of its incident edges is forced. Similarly, it is called unforced if no forced edge is incident to it. A Hamiltonian cycle in $G$ is called a tour if it passes through all the forced edges in $F$. Under these circumstances, the forced TSP requests to find a minimum cost tour of an instance $(G, F)$, or determine that none exists.

Throughout this paper, we assume that the maximum degree of a vertex in $G$ is at most 5. We denote a forced (resp., unforced) vertex of degree $i$ as a type fi vertex (resp., ui vertex). We are interested in six types of vertices in an instance of $(G, F)$, namely, $\mathrm{u} 5, \mathrm{f} 5, \mathrm{u} 4, \mathrm{f} 4, \mathrm{u} 3$ and f3-vertices. Let $V_{\mathrm{f} i}\left(\right.$ resp., $\left.V_{\mathrm{u} i}\right), i=3,4,5$, denote the set of fi-vertices (resp., u $i$-vertices) in $(G, F)$.

\subsection{A Polynomial-Space Branching Algorithm}

Our algorithm contains two major steps which are repeated recursively. In the first step, the algorithm applies reduction rules until no further reduction is possible. Once a feasible instance which cannot be further reduced has been obtained, the algorithm branches in search of a solution by either including a chosen unforced edge $e$ into $F$, force $(e)$, or excluding it from $E(G)$, delete $(e)$. The choice of the edge $e$ to branch on is based on a set of branching rules. By applying a branching operation, the algorithm generates two new instances, called branches.

\subsubsection{Reduction Rules}

Reduction is the process of transforming an instance to a smaller instance while preserving its optimality. It takes polynomial time to obtain a solution of an original instance from a solution of a smaller instance that has been obtained by a reduction procedure from the original instance.

Not all instances of the forced TSP have a tour. If an instance has no tour, we call it infeasible. Observation 1 gives two sufficient conditions for an instance to be infeasible, as observed by Rubin [14].

Observation 1 An instance $(G, F)$ is infeasible if one of the following conditions holds.

(i) $d(v) \leq 1$ for some vertex $v \in V(G)$, and

(ii) $d_{F}(v) \geq 3$ for some vertex $v \in V(G)$.

In this paper, there are two reduction rules which are applied following any branching operation, following Observation 2.

Observation 2 Each of the following reductions preserves 
the feasibility and a minimum cost tour of an instance $(G, F)$.

(i) If $d(v)=2$ for a vertex $v$, then add to $F$ any unforced edge incident to the vertex $v$; and

(ii) If $d(v)>2$ and $d_{F}(v)=2$ for a vertex $v$, then remove from $G$ any unforced edge incident to the vertex $v$.

An instance $(G, F)$ which does not satisfy any of the conditions in Observation 1 and Observation 2 is called a reduced instance.

Based on Observation 1 and Observation 2, we can form a reduction procedure that given an instance $(G, F)$ of the forced TSP returns a reduced instance, or a message for the infeasibility of $(G, F)$, which for convenience we can assume that evaluates to $\infty$. For completeness, we describe such a procedure as Algorithm $\operatorname{Red}(G, F)$.

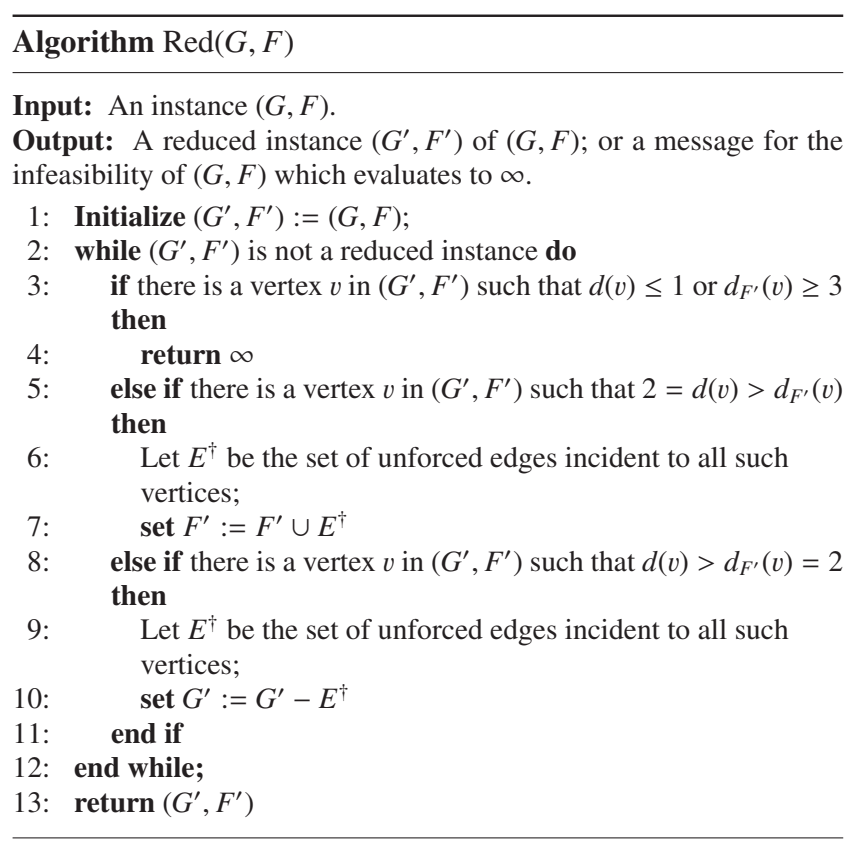

\subsubsection{Branching Rules}

To describe our branching algorithm, let $(G, F)$ be a reduced instance such that the maximum degree of $G$ is at most 5. In $(G, F)$, an unforced edge $e=v t$ incident to a vertex $v$ of degree 5 is called optimal, if it satisfies a condition c- $i$ below with minimum index $i$, over all unforced edges $v t$ in $(G, F)$. We refer to the following conditions for choosing an optimal edge to branch on, c-1 to c-15, as the branching rules.

(c-1) $v \in V_{\mathrm{f} 5}$ and $t \in N_{U}\left(v ; V_{\mathrm{f} 3}\right)$ such that $N_{U}(v) \cap N_{U}(t)=\emptyset$;

(c-2) $v \in V_{\mathrm{f} 5}$ and $t \in N_{U}\left(v ; V_{\mathrm{f} 3}\right)$ such that $N_{U}(v) \cap N_{U}(t) \neq \emptyset$;

(c-3) $v \in V_{\mathrm{f} 5}$ and $t \in N_{U}\left(v ; V_{\mathrm{u} 3}\right)$;

(c-4) $v \in V_{\mathrm{f} 5}$ and $t \in N_{U}\left(v ; V_{\mathrm{f} 4}\right)$ such that $N_{U}(v) \cap N_{U}(t)=\emptyset$;

(c-5) $v \in V_{\mathrm{f} 5}$ and $t \in N_{U}\left(v ; V_{\mathrm{f} 4}\right)$ such that $N_{U}(v) \cap N_{U}(t) \neq \emptyset$;

(I) $\left|N_{U}(v) \cap N_{U}(t)\right|=1$; and

(II) $\left|N_{U}(v) \cap N_{U}(t)\right|=2$;

(c-6) $v \in V_{\mathrm{f} 5}$ and $t \in N_{U}\left(v ; V_{\mathrm{u} 4}\right)$;

(c-7) $v \in V_{\mathrm{f} 5}$ and $t \in N_{U}\left(v ; V_{\mathrm{f} 5}\right)$ such that $N_{U}(v) \cap N_{U}(t)=\emptyset$;

(c-8) $v \in V_{\mathrm{f} 5}$ and $t \in N_{U}\left(v ; V_{\mathrm{f} 5}\right)$ such that $N_{U}(v) \cap N_{U}(t) \neq \emptyset$;

(I) $\left|N_{U}(v) \cap N_{U}(t)\right|=1$;

(II) $\left|N_{U}(v) \cap N_{U}(t)\right|=2$; and

(III) $\left|N_{U}(v) \cap N_{U}(t)\right|=3$;

(c-9) $v \in V_{\mathrm{f} 5}$ and $t \in N_{U}\left(v ; V_{\mathrm{u} 5}\right)$;

(c-10) $v \in V_{\mathrm{u} 5}$ and $t \in N_{U}\left(v ; V_{\mathrm{f} 3}\right)$ such that $N_{U}(v) \cap N_{U}(t) \neq \emptyset$;
Assume without loss of generality that $N_{U}(v) \cap N_{U}(t)=$ $\left\{t_{2}\right\}$.

(I) $t_{2}$ is an f3-vertex,

(II) $t_{2}$ is a u3-vertex,

(III) $t_{2}$ is an f4-vertex,

(IV) $t_{2}$ is a u4-vertex, and

(V) $t_{2}$ is a u5-vertex.

(c-11) $v \in V_{\mathrm{u} 5}$ and $t \in N_{U}\left(v ; V_{\mathrm{f} 3}\right)$, such that $N_{U}(v) \cap N_{U}(t)=\emptyset$;

(c-12) $v \in V_{\mathrm{u} 5}$ and $t \in N_{U}\left(v ; V_{\mathrm{u} 3}\right)$;

(c-13) $v \in V_{\mathrm{u} 5}$ and $t \in N_{U}\left(v ; V_{\mathrm{f} 4}\right)$;

(c-14) $v \in V_{\mathrm{u} 5}$ and $t \in N_{U}\left(v ; V_{\mathrm{u} 4}\right)$; and

(c-15) $v \in V_{\mathrm{u} 5}$ and $t \in N_{U}\left(v ; V_{\mathrm{u} 5}\right)$.

The collective set of branching rules are illustrated in Fig. 1. The given list of branching rules is exhaustive, in the sense that each possible combination of a degree 5 vertex and choice for its neighbor are included.

For convenience in the analysis of the algorithm, cases c-5 and c- 8 have been subdivided into sub-cases according to the cardinality of the intersection of the neighborhoods of the vertices $v$ and $t$. Intersections of lower cardinality take precedence over higher ones. Case c-10 has been subdivided into sub-cases according to the type of the unique vertex in the neighborhood intersection of vertices $v$ and $t$.

Given a reduced instance $(G, F)$, our algorithm checks whether there exists a vertex of degree 5. If it does, then the algorithm chooses an optimal edge according to the branching rules, and branches into two recursive branches; one branch that includes the selected edge into the set of forced edges, and the other branch removes the edge from the set of edges of the graph $G$. If there does not exist a vertex of degree 5 in the graph $G$ of the given instance, then we can call a polynomial space exact algorithm for the TSP that is specialized for degree-4 graphs, e.g., the algorithm due to Xiao and Nagamochi [16]. Our branching algorithm is described in Algorithm tsp5 $(G, F)$.

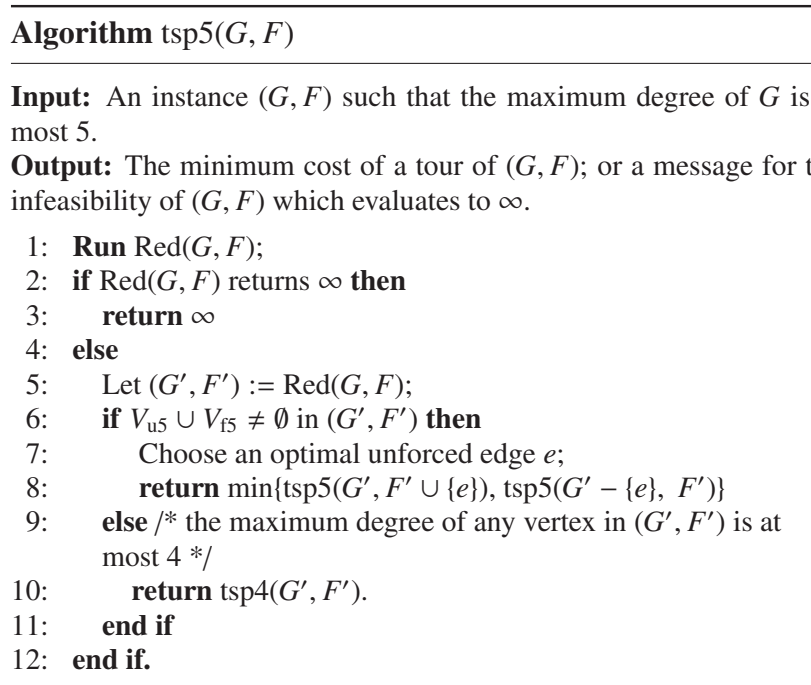

Note: The input and output of algorithm tsp $4(G, F)$ are as follows:

Input: An instance $(G, F)$ such that the maximum degree of $G$ is at most 4 .

Output: The minimum cost of a tour of $(G, F)$; or a message for the infeasibility of $(G, F)$ which evaluates to $\infty$. 


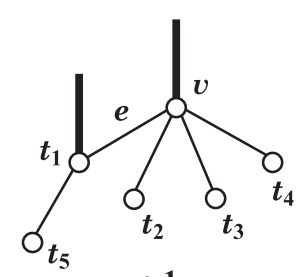

c-1

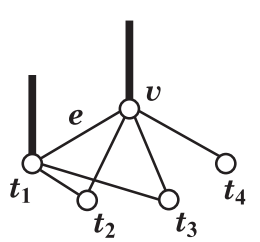

c-5(II)

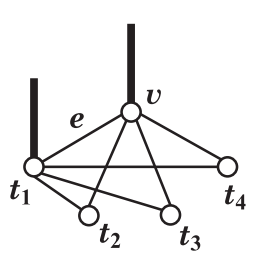

c-8(III)

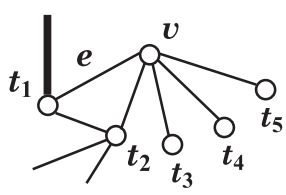

c-10(IV)

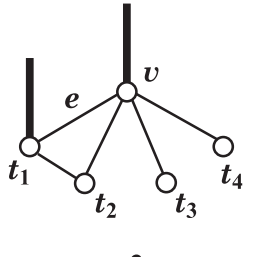

c-2<smiles>COC(C)(C)O</smiles>

c-6

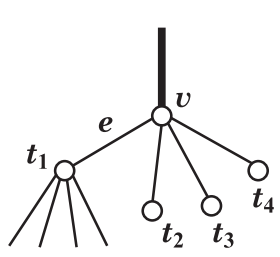

c-9
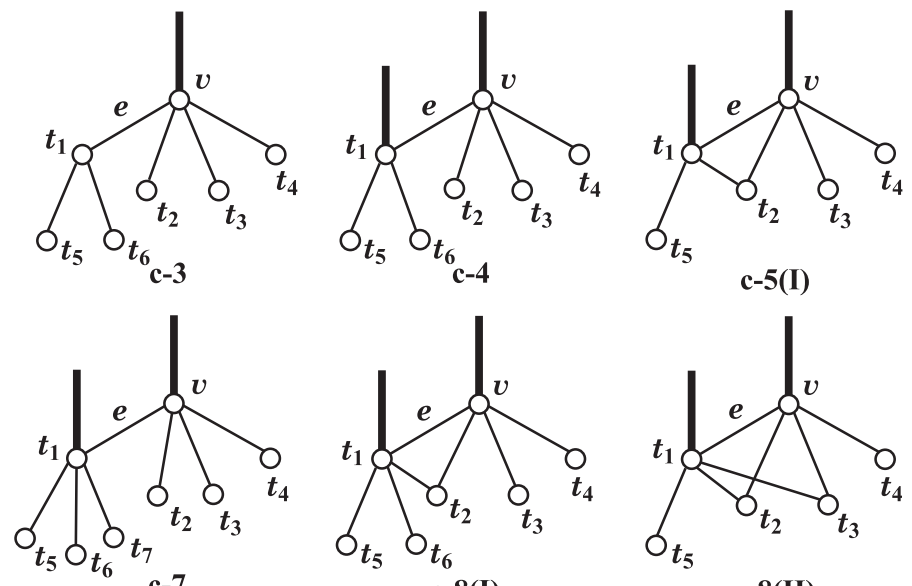

c-8(I)

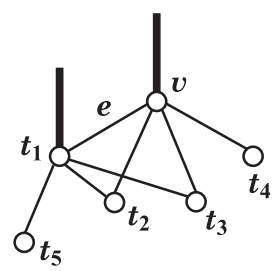

c-8(II)

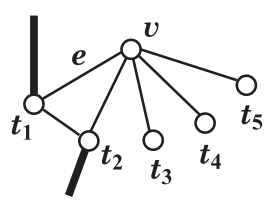

c-10(I)

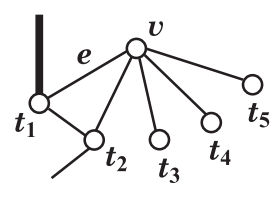

c-10(II)

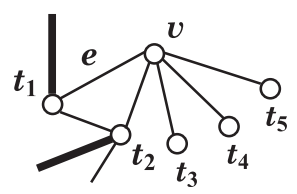

c-10(III)

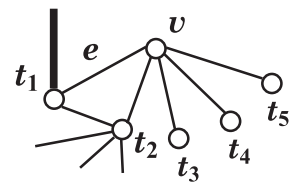

c-10(V)

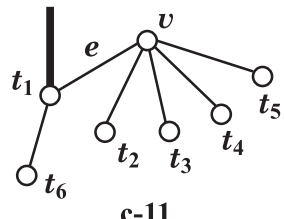

c-11

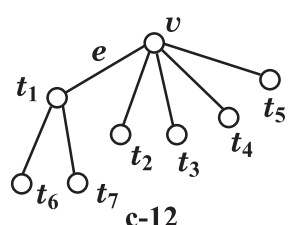

c-12

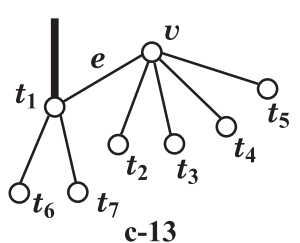

c-13

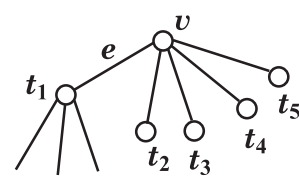

c-14

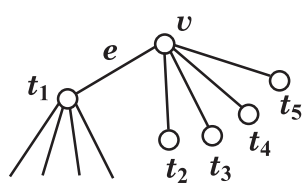

c-15

Fig. 1 Illustration of the Branching Rules.

\section{Analysis}

\subsection{Analysis Framework}

To effectively analyze the running time of our branching algorithm, we use the measure-and-conquer method as introduced by Fomin et al. [6]. Given an instance $I=(G, F)$ of the forced TSP, we assign a nonnegative weight $\omega(v)$ to each vertex $v \in V(G)$ according to its type. To this effect, we set a non-negative vertex weight function $\omega: V \rightarrow \mathbb{R}_{+}$in the graph $G$, and we use the sum of weights of all vertices in the graph as the measure $\mu(I)$ of instance $I$, that is,

$$
\mu(I) \triangleq \sum_{v \in V(G)} \omega(v)
$$

It is important for the analysis to find a measure which satisfies the following properties:

(i) $\mu(I)=0$ if and only if $I$ can be solved in polynomial time; and

(ii) If $I^{\prime}$ is a sub-instance of $I$ obtained through a reduction or a branching operation, then $\mu\left(I^{\prime}\right) \leq \mu(I)$.
We call a measure $\mu$ satisfying conditions (i) and (ii) above a proper measure.

A branching algorithm typically comprises multiple branching rules. We perform the time analysis of the branching algorithm via appropriately constructed recurrences over the measure $\mu=\mu(I)$ of an instance $I=(G, F)$, for each branching rule of the algorithm. Let $T(\mu)$ denote the number of nodes in the search tree generated by a branching rule when invoked on the instance $I$ with measure $\mu$. For $k \geq 2$, let $I^{1}, I^{2}, \ldots, I^{k}$ be the instances obtained from $I$ by the branching operation, and for $i=1,2, \ldots, k$, let $t_{i} \leq \mu(I)-\mu\left(I^{i}\right)$ be lower bounds on the amounts of decrease in the measure. We call $\left(t_{1}, t_{2}, \ldots, t_{k}\right)$ a branching vector of the branching operation, and this implies the linear recurrence

$$
T(\mu) \leq T\left(\mu-t_{1}\right)+T\left(\mu-t_{2}\right)+\cdots+T\left(\mu-t_{k}\right) .
$$

To evaluate the performance of this branching vector, we can use any standard method for linear recurrence relations. In fact, it is known that $T(\mu)$ is of the form $O\left(\tau^{\mu}\right)$, where $\tau$ is the unique positive real root of the function $f(x)=1-\left(x^{-t_{1}}+x^{-t_{2}}+\cdots+x^{-t_{k}}\right)$. The value $\tau$ is called the branching factor of the branching vector 
$\left(t_{1}, t_{2}, \ldots, t_{k}\right)$. The running time of the algorithm is determined by considering the worst branching factor over all branching vectors generated by all of the branching rules of the algorithm.

For further details justifying this approach, as well as a solid introduction to branching algorithms, the reader is referred to the book of Fomin and Kratsch [7].

\subsection{Weight Setting}

In order to obtain a measure which will naturally give a running time bound as a function of the size of a TSP instance, we require that the weight of each vertex be at most 1 . In what follows, we examine some necessary constraints which the vertex weights should satisfy in order for us to obtain a proper measure.

For each $i \in\{3,4,5\}$, we denote by $w_{i}$ the weight of a u $i$-vertex, and by $w_{i}^{\prime}$ the weight of an fi-vertex. The conditions for a proper measure require that the measure of an instance obtained through a reduction operation or a branching operation be not greater than the measure of the original instance. Thus, the vertex weights should satisfy the following relations:

$$
\begin{aligned}
& w_{5} \leq 1, \\
& w_{i}^{\prime} \leq w_{i}, \quad i=3,4,5, \\
& w_{3} \leq w_{4} \leq w_{5}, \text { and } \\
& w_{3}^{\prime} \leq w_{4}^{\prime} \leq w_{5}^{\prime} .
\end{aligned}
$$

The vertex weight for vertices of degree less than 3 is set to be 0 .

Lemma 1 states that given $\operatorname{Algorithm} \operatorname{Red}(G, F)$ and Algorithm $\operatorname{tsp} 5(G, F)$, setting vertex weights which satisfy the conditions of Eqs. (4) to (6) is sufficient to obtain a proper measure. This lemma has been proved by Md Yunos et al. [13].

Lemma 1 If the weights of vertices are chosen as in Eqs. (4) to (6), then the measure $\mu(I)$ never increases as a result of the reduction or the branching operations of $\operatorname{Algorithm} \operatorname{Red}(G, F)$ and Algorithm tsp5 $(G, F)$.

Proof. Let $I=(G, F)$ be a given instance of the forced TSP. Due to our definition of the measure $\mu(I)$ of Eq. (1), it suffices to show that none of the individual vertex weights will increase as a result of a reduction operation or a branching operation in Algorithm $\operatorname{Red}(G, F)$ and Algorithm tsp5 $(G, F)$.

The branching rules state that for an unforced edge $e$ in $E(G) \backslash$ $F$, two sub-instances are generated by either setting $F:=F \cup\{e\}$, termed force $(e)$, or by setting $G:=G-\{e\}$, termed delete $(e)$. We bring to the reader's attention that a reduction operation is in fact a repeated application of the above two steps, force $(e)$ or delete $(e)$, for some unforced edge $e$, identified by the conditions in Observation 2. Therefore, we proceed with analyzing the effects of applying each of the force $(e)$ or the delete $(e)$ operations.

Let $e=u v$ be an unforced edge to which one of the force $(e)$ or delete $(e)$ operations will be applied. Without loss of generality, we observe the effect of the operation on the vertex weight $\omega(v)$.

In the case where operation force $(e)$ is applied, the following cases may arise.

- If $v$ is an unforced vertex, then $v$ will become forced. By Eq. (4), the weight $\omega(v)$ will not increase.

- If $v$ is a forced vertex, then $\omega(v)$ will become 0 ; and

- If $d_{F}(v) \geq 2$, then by Observation 1 the instance will become infeasible.

On the other hand, if operation $\operatorname{delete}(e)$ is applied, then we observe the following cases.

- If $v$ is either forced or unforced, and $d(v) \geq 3$, then the degree of $v$ will decrease by one, and by Eqs. (5) and (6), $\omega(v)$ will not increase; and

- If $v$ is either forced or unforced, and $d(v) \leq 2$, then by Observation 1 the instance will become infeasible.

Following the above observations, we conclude that the complete measure $\mu(I)$ of a given instance $I=(G, F)$ of the forced TSP will not increase as a result of the reduction and the branching operations in $\operatorname{Algorithm~} \operatorname{Red}(G, F)$ and Algorithm tsp5 $(G, F)$.

To simplify some arguments and the list of the branching vectors we are about to derive, we introduce the following notation:

$$
\begin{aligned}
& \Delta_{i} \triangleq w_{i}-w_{i}^{\prime}, \quad 3 \leq i \leq 5 \\
& \Delta_{i, j} \triangleq w_{i}-w_{j}, \quad 3 \leq j<i \leq 5, \text { and } \\
& \Delta_{i, j}^{\prime} \triangleq w_{i}^{\prime}-w_{j}^{\prime}, \quad 3 \leq j<i \leq 5,
\end{aligned}
$$

further,

$$
\begin{aligned}
& m_{1} \triangleq \min \left\{w_{3}^{\prime}, w_{3}, \Delta_{4,3}^{\prime}, \Delta_{4,3}, \Delta_{5,4}^{\prime}, \Delta_{5,4}\right\}, \\
& m_{2} \triangleq \min \left\{w_{3}, \Delta_{4,3}^{\prime}, \Delta_{4,3}, \Delta_{5,4}^{\prime}, \Delta_{5,4}\right\}, \\
& m_{3} \triangleq \min \left\{w_{3}^{\prime}, \Delta_{3}, w_{4}^{\prime}, \Delta_{4}, w_{5}^{\prime}, \Delta_{5}\right\}, \\
& m_{4} \triangleq \min \left\{\Delta_{4,3}^{\prime}, \Delta_{4,3}, \Delta_{5,4}^{\prime}, \Delta_{5,4}\right\}, \\
& m_{5} \triangleq \min \left\{w_{4}^{\prime}, w_{4}, \Delta_{5,3}^{\prime}, \Delta_{5,3}\right\}, \\
& m_{6} \triangleq \min \left\{\Delta_{4,3}, \Delta_{5,4}^{\prime}, \Delta_{5,4}\right\}, \\
& m_{7} \triangleq \min \left\{\Delta_{5,4}^{\prime}, \Delta_{5,4}\right\}, \\
& m_{8} \triangleq \min \left\{\Delta_{5,3}^{\prime}, \Delta_{5,3}\right\}, \\
& m_{9} \triangleq \min \left\{w_{3}^{\prime}, \Delta_{3}, w_{4}^{\prime}, \Delta_{4}, \Delta_{5}\right\}, \\
& m_{10} \triangleq \min \left\{w_{3}^{\prime}, w_{3}, \Delta_{4,3}^{\prime}, \Delta_{4,3}, \Delta_{5,4}\right\}, \text { and } \\
& m_{11} \triangleq \min \left\{w_{3}^{\prime}, w_{3}, w_{4}^{\prime}, w_{4}-w_{3}^{\prime}, w_{5}-w_{4}^{\prime}\right\} .
\end{aligned}
$$

\subsection{Main Results}

We bring to the reader's attention the fact that the number $n$ of vertices in the graph $G$ remains unmodified throughout the process of the reduction and the branching operations. In addition to seeking a proper measure, we also require that the weight of each vertex be at most 1 , and therefore, the measure $\mu(I)$ will not be greater than the number $n$ of vertices in $G$. As a consequence, a running time bound as a function of the measure $\mu(I)$ implies the same running time bound as a function of $n$. The weight assigned to each vertex type plays an important role, since the value of the branching factor depends solely on these weights.

Let the vertex weight function $\omega(v)$ be chosen as follows:

$$
\omega(v) \triangleq\left\{\begin{array}{cl}
w_{5}=1.000000 & \text { for a u5-vertex } v \\
w_{5}^{\prime}=0.714884 & \text { for an f5-vertex } v \\
w_{4}=0.653197 & \text { for a u4-vertex } v \\
w_{4}^{\prime}=0.368082 & \text { for an f4-vertex } v \\
w_{3}=0.306395 & \text { for a u3-vertex } v \\
w_{3}^{\prime}=0.160872 & \text { for an f3-vertex } v \\
0 & \text { otherwise. }
\end{array}\right.
$$

The vertex weight function $\omega(v)$ given in Eq. (18) is obtained 


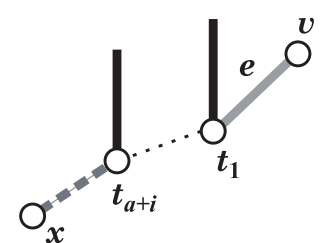

(a) force $(e)$

: forced edges the reduction rules

$\ldots \ldots$ : newly deleted edges by the reduction rules $-m:$ : newly forced edges by

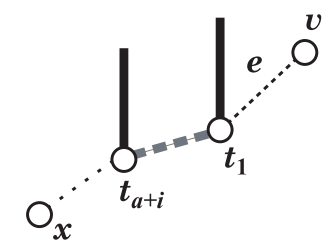

(b) $\operatorname{delete}(e)$

: newly forced edges by the branching operation : newly deleted edges by the branching operation
Fig. 2 Illustration of forced and deleted edges by the branching operation and reduction rules for an $\mathrm{f} 3$ vertex.

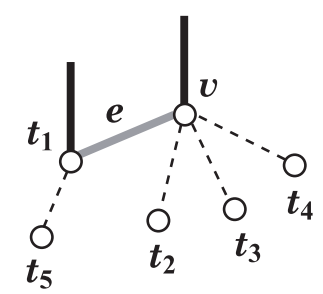

(a) force $\left(v t_{1}\right)$ in c-1

: unforced edges forced edges

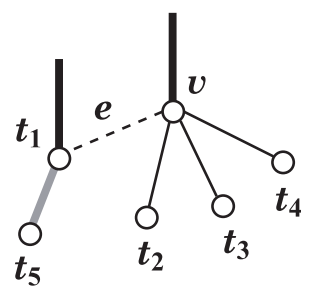

(b) delete $\left(v t_{1}\right)$ in $\mathrm{c}-1$ : newly deleted edges newly forced edges
Fig. 3 Illustration of branching rule c-1, where vertex $v \in V_{\mathrm{f} 5}$ and vertex $t_{1} \in N_{U}\left(v ; V_{\mathrm{f} 3}\right)$ such that $N_{U}(v) \cap N_{U}\left(t_{1}\right)=\emptyset$.

as a solution to a quasiconvex program, according to the method introduced by Eppstein [4]. All the branching vectors are in fact constraints in the quasiconvex program.

Lemma 2 If the vertex weight function $\omega(v)$ is set as in Eq. (18), then each branching operation in Algorithm tsp5( $G, F)$ has a branching factor not greater than 2.349978.

A proof of Lemma 2 will be derived analytically in the several subsections which follow. From the lemma, we get our main result as stated in Theorem 1.

Theorem 1 The TSP in an $n$-vertex graph $G$ with maximum degree 5 can be solved in $O^{*}\left(2.3500^{n}\right)$ time and polynomial space.

In the remainder of the analysis, for an optimal edge $e=v t_{1}$, we denote $N_{U}(v)$ by $\left\{t_{1}, t_{2}, \ldots, t_{a}\right\}, a=d_{U}(v)$, and $N_{U}\left(t_{1}\right) \backslash\{v\}$ by $\left\{t_{a+1}, t_{a+2}, \ldots, t_{a+b}\right\}, b=d_{U}\left(t_{1}\right)-1$. If $N_{U}(v) \cap N_{U}\left(t_{1}\right) \neq \emptyset$, then let $c=\left|N_{U}(v) \cap N_{U}\left(t_{1}\right)\right|$, and we can assume without loss of generality that $t_{1+i}=t_{a+i}$ for $i=1,2, \ldots, c$.

If $N_{U}(v) \cap N_{U}\left(t_{1}\right)=\emptyset$, and there exists an f3-vertex $t_{a+i}$ in $N_{U}\left(t_{1}\right) \backslash\{v\}$, let $x \in N_{U}\left(t_{a+i}\right) \backslash\left\{t_{1}\right\}$. We see that the choice of vertex $x$ is unique, because $t_{a+i}$ is of type $\mathrm{f} 3$ and $\left|N_{U}\left(t_{a+i}\right) \backslash\left\{t_{1}\right\}\right|=1$. This vertex $x$ plays a key role in our analysis, namely in cases c-1 and c-11, as shown in Fig. 2 .

\subsection{Branching on Edges Around f5-vertices (Branching Rules c-1 to c-9)}

This section derives branching vectors for the branching operations on an optimal edge $e=v t_{1}$, incident to an f5-vertex $v$, distinguishing nine cases for conditions c-1 to c-9.

Case c-1. There exist vertices $v \in V_{\mathrm{f} 5}$ and $t_{1} \in N_{U}\left(v ; V_{\mathrm{f} 3}\right)$ such that $N_{U}(v) \cap N_{U}\left(t_{1}\right)=\emptyset$ (see Fig. 3): We branch on edge $v t_{1}$. Note that $N_{U}\left(t_{1}\right) \backslash\{v\}=\left\{t_{5}\right\}$.
In the branch of force $\left(v t_{1}\right)$, the edge $v t_{1}$ will be added to $F^{\prime}$ by the branching operation, and edges $v t_{2}, v t_{3}, v t_{4}$ and $t_{1} t_{5}$ will be deleted from $G^{\prime}$ by the reduction rules. Both $v$ and $t_{1}$ will become vertices of degree 2. From Eq. (18), the weight of vertices of degree 2 is 0 . So, the weight of vertex $v$ decreases by $w_{5}^{\prime}$ and the weight of vertex $t_{1}$ decreases by $w_{3}^{\prime}$.

In the branch of delete $\left(v t_{1}\right)$, the edge $v t_{1}$ will be deleted from $G^{\prime}$ by the branching operation, and the edge $t_{1} t_{5}$ will be added to $F^{\prime}$ by the reduction rules. The weight of vertex $v$ decreases by $\Delta_{5,4}^{\prime}$ and the weight of vertex $t_{1}$ decreases by $w_{3}^{\prime}$.

There are two cases for the vertex $t_{5} ; 1$ ) vertex $t_{5}$ is of type $\mathrm{f} 3$, and 2) otherwise. We will analyze these two cases separately for each branch force $\left(v t_{1}\right)$ and delete $\left(v t_{1}\right)$.

First, we analyze the case where the vertex $t_{5}$ is an $\mathrm{f} 3$-vertex (see Fig. 2). Recall that in such a case we denote by $x$ the unique vertex in $N_{U}\left(t_{5}\right) \backslash\left\{t_{1}\right\}$. There are two cases for the vertex $x$; a) vertex $x \notin N_{U}(v)$, and b) vertex $x \in N_{U}(v)$.

First, we analyze the case where the vertex $x \notin N_{U}(v)$. In the branch of force $\left(v t_{1}\right)$, the edge $x t_{5}$ will be added to $F^{\prime}$ by the reduction rules. Hence, each of the vertices $t_{2}, t_{3}$ and $t_{4}$ must be one of types f3, u3, f4, u4, f5, and a u5-vertex, and each of their weights decreases by at least $m_{1}=\min \left\{w_{3}^{\prime}, w_{3}, \Delta_{4,3}^{\prime}, \Delta_{4,3}, \Delta_{5,4}^{\prime}, \Delta_{5,4}\right\}$. The weight of vertex $t_{5}$ decreases by $w_{3}^{\prime}$. If the vertex $x$ is an $\mathrm{f} 3$-vertex (resp., u3, f4, u4, f5, and a u5-vertex), then the weight decrease $\alpha_{1}$ of vertex $x$ will be $w_{3}^{\prime}$ (resp., $\Delta_{3}, w_{4}^{\prime}, \Delta_{4}, w_{5}^{\prime}$, and $\Delta_{5}$ ). Thus, the total weight decrease for this case in the branch of force $\left(v t_{1}\right)$ is at least $w_{5}^{\prime}+w_{3}^{\prime}+3 m_{1}+w_{3}^{\prime}+\alpha_{1}$.

In the branch of delete $\left(v t_{1}\right)$, the edge $x t_{5}$ will be deleted from $G^{\prime}$ by the reduction rules. Hence, the weight of vertex $t_{5}$ decreases by $w_{3}^{\prime}$. If the vertex $x$ is an f3-vertex (resp., u3, f4, $\mathrm{u} 4$, f5, and a u5-vertex), then the weight decrease $\beta_{1}$ of vertex $x$ will be $w_{3}^{\prime}$ (resp., $w_{3}, \Delta_{4,3}^{\prime}, \Delta_{4,3}, \Delta_{5,4}^{\prime}$, and $\Delta_{5,4}$ ). Thus, the total weight decrease for this case in the branch of delete $\left(v t_{1}\right)$ is at least $w_{5}^{\prime}-w_{4}^{\prime}+w_{3}^{\prime}+w_{3}^{\prime}+\beta_{1}$.

As a result, for the ordered pair $\left(\alpha_{1}, \beta_{1}\right)$ taking values in $\left\{\left(w_{3}^{\prime}, w_{3}^{\prime}\right),\left(\Delta_{3}, w_{3}\right),\left(w_{4}^{\prime}, \Delta_{4,3}^{\prime}\right),\left(\Delta_{4}, \Delta_{4,3}\right),\left(w_{5}^{\prime}, \Delta_{5,4}^{\prime}\right),\left(\Delta_{5}, \Delta_{5,4}\right)\right\}$, we get the following six branching vectors:

$$
\left(w_{5}^{\prime}+2 w_{3}^{\prime}+3 m_{1}+\alpha_{1}, w_{5}^{\prime}-w_{4}^{\prime}+2 w_{3}^{\prime}+\beta_{1}\right) .
$$

Second, we analyze the case where the vertex $x \in N_{U}(v)$. Without loss of generality, we assume that $x$ is $t_{2}$. In the branch of force $\left(v t_{1}\right)$, the edge $t_{2} t_{5}$ will be added to $F^{\prime}$ by the reduction rules. Each of the vertices $t_{3}$ and $t_{4}$ must be one of types f3, u3, f4, u4, $\mathrm{f} 5$, and a u5-vertex, and each of their weights decreases by at least $m_{1}=\min \left\{w_{3}^{\prime}, w_{3}, \Delta_{4,3}^{\prime}, \Delta_{4,3}, \Delta_{5,4}^{\prime}, \Delta_{5,4}\right\}$. The weight of vertex $t_{5}$ decreases by $w_{3}^{\prime}$. If the vertex $t_{2}$ is an f3-vertex (resp., u3, f4, u4, $\mathrm{f} 5$, and a u5-vertex), then the weight decrease $\alpha_{2}$ of vertex $x$ will be $w_{3}^{\prime}$ (resp., $w_{3}, w_{4}^{\prime}, w_{4}-w_{3}^{\prime}, w_{5}^{\prime}$, and $w_{5}-w_{4}^{\prime}$ ). Thus, the total weight decrease for this case in the branch of force $\left(v t_{1}\right)$ is at least $w_{5}^{\prime}+w_{3}^{\prime}+2 m_{1}+w_{3}^{\prime}+\alpha_{2}$.

In the branch of delete $\left(v t_{1}\right)$, the edge $t_{2} t_{5}$ will be deleted from $G^{\prime}$ by the reduction rules. Hence, the weight of vertex $t_{5}$ decreases by $w_{3}^{\prime}$. If the vertex $t_{2}$ is an f3-vertex (resp., u3, f4, $\mathrm{u} 4$, f5, and a u5-vertex), then the weight decrease $\beta_{2}$ of vertex $x$ will be $w_{3}^{\prime}$ (resp., $w_{3}, \Delta_{4,3}^{\prime}, \Delta_{4,3}, \Delta_{5,4}^{\prime}$, and $\Delta_{5,4}$ ). Thus, the total weight decrease for this case in the branch of delete $\left(v t_{1}\right)$ is at 


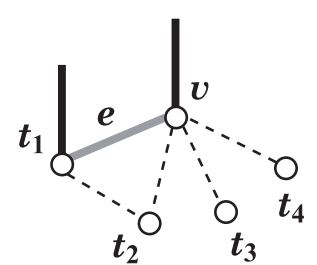

(a) force $\left(v t_{1}\right)$ in c-2

: unforced edges forced edges

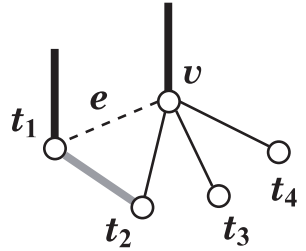

(b) $\operatorname{delete}\left(v t_{1}\right)$ in c-2

: newly deleted edges : newly forced edges
Fig. 4 Illustration of branching rule c-2, where vertex $v \in V_{\mathrm{f} 5}$ and vertex $t_{1} \in N_{U}\left(v ; V_{\mathrm{f} 3}\right)$ such that $N_{U}(v) \cap N_{U}\left(t_{1}\right)=\left\{t_{2}\right\}$.

least $w_{5}^{\prime}-w_{4}^{\prime}+w_{3}^{\prime}+w_{3}^{\prime}+\beta_{2}$.

As a result, for the ordered pair $\left(\alpha_{2}, \beta_{2}\right)$ taking values in $\left\{\left(w_{3}^{\prime}, w_{3}^{\prime}\right),\left(w_{3}, w_{3}\right),\left(w_{4}^{\prime}, \Delta_{4,3}^{\prime}\right),\left(w_{4}-w_{3}^{\prime}, \Delta_{4,3}\right),\left(w_{5}^{\prime}, \Delta_{5,4}^{\prime}\right),\left(w_{5}-\right.\right.$ $\left.\left.w_{4}^{\prime}, \Delta_{5,4}\right)\right\}$, we get the following six branching vectors:

$$
\left(w_{5}^{\prime}+2 w_{3}^{\prime}+2 m_{1}+\alpha_{2}, w_{5}^{\prime}-w_{4}^{\prime}+2 w_{3}^{\prime}+\beta_{2}\right) .
$$

Next, we examine the case where the vertex $t_{5}$ is not an f3vertex. In the branch of force $\left(v t_{1}\right)$, if the vertex $t_{5}$ is a u3-vertex (resp., f4, u4, f5, and a u5-vertex), then the weight decrease $\alpha_{3}$ of vertex $t_{5}$ will be $w_{3}$ (resp., $\Delta_{4,3}^{\prime}, \Delta_{4,3}, \Delta_{5,4}^{\prime}$, and $\Delta_{5,4}$ ). Thus, the total weight decrease for this case in the branch of force $\left(v t_{1}\right)$ is at least $w_{5}^{\prime}+w_{3}^{\prime}+3 m_{1}+\alpha_{3}$.

In the branch of delete $\left(v t_{1}\right)$, if the vertex $t_{5}$ is a u3-vertex (resp., $\mathrm{f} 4, \mathrm{u} 4, \mathrm{f} 5$, and a $\mathrm{u} 5$-vertex), then the weight decrease $\beta_{3}$ of vertex $t_{5}$ will be $\Delta_{3}$ (resp., $w_{4}^{\prime}, \Delta_{4}, w_{5}^{\prime}$, and $\Delta_{5}$ ). Thus, the total weight decrease for the case where $t_{5}$ is not an f3-vertex in the branch of $\operatorname{delete}\left(v t_{1}\right)$ is at least $w_{5}^{\prime}-w_{4}^{\prime}+w_{3}^{\prime}+\beta_{3}$.

As a result, for the ordered pair $\left(\alpha_{3}, \beta_{3}\right)$ taking values in $\left\{\left(w_{3}, \Delta_{3}\right),\left(\Delta_{4,3}^{\prime}, w_{4}^{\prime}\right),\left(\Delta_{4,3}, \Delta_{4}\right),\left(\Delta_{5,4}^{\prime}, w_{5}^{\prime}\right),\left(\Delta_{5,4}, \Delta_{5}\right)\right\}$, we get the following five branching vectors:

$$
\left(w_{5}^{\prime}+w_{3}^{\prime}+3 m_{1}+\alpha_{3}, w_{5}^{\prime}-w_{4}^{\prime}+w_{3}^{\prime}+\beta_{3}\right)
$$

Case c-2. Case c-1 is not applicable, and there exist vertices $v \in V_{\mathrm{f} 5}$ and $t_{1} \in N_{U}\left(v ; V_{\mathrm{f} 3}\right)$ such that $N_{U}(v) \cap N_{U}\left(t_{1}\right)=\left\{t_{2}\right\}$ (see Fig. 4): We branch on edge $v t_{1}$.

In the branch of force $\left(v t_{1}\right)$, the edge $v t_{1}$ will be added to $F^{\prime}$ by the branching operation, and edges $v t_{2}, v t_{3}, v t_{4}$ and $t_{1} t_{2}$ will be deleted from $G^{\prime}$ by the reduction rules. So, the weight of vertex $v$ decreases by $w_{5}^{\prime}$, and the weight of vertex $t_{1}$ decreases by $w_{3}^{\prime}$. Each of the vertices $t_{3}$ and $t_{4}$ must be one of types $\mathrm{f} 3, \mathrm{u} 3, \mathrm{f} 4, \mathrm{u} 4$, f5, and a u5-vertex, and each of their weights decreases by at least $m_{1}=\min \left\{w_{3}^{\prime}, w_{3}, \Delta_{4,3}^{\prime}, \Delta_{4,3}, \Delta_{5,4}^{\prime}, \Delta_{5,4}\right\}$.

If the vertex $t_{2}$ is an $\mathrm{f} 3$ or a u3-vertex, after performing the branching operation, $t_{2}$ becomes a vertex of degree 1 . From $\mathrm{Ob}-$ servation 1 , case (i), this is infeasible, and the algorithm will return $\infty$ and terminate. Otherwise, if the vertex $t_{2}$ is an f4-vertex (resp., u4, f5, and a u5-vertex), then the weight decrease $\alpha_{4}$ of vertex $t_{2}$ will be $w_{4}^{\prime}$ (resp., $w_{4}, \Delta_{5,3}^{\prime}$, and $\Delta_{5,3}$ ). Thus, the total weight decrease in the branch of force $\left(v t_{1}\right)$ is at least $w_{5}^{\prime}+w_{3}^{\prime}+2 m_{1}+\alpha_{4}$.

In the branch of delete $\left(v t_{1}\right)$, the edge $v t_{1}$ will be deleted from $G^{\prime}$ by the branching operation, and the edge $t_{1} t_{2}$ will be added to $F^{\prime}$ by the reduction rules. So, the weights of vertices $v$ and $t_{1}$ decrease by $\Delta_{5,4}^{\prime}$ and $w_{3}^{\prime}$, respectively. If the vertex $t_{2}$ is an f4-vertex (resp., u4, f5, and a u5-vertex), then the weight decrease $\beta_{4}$ of

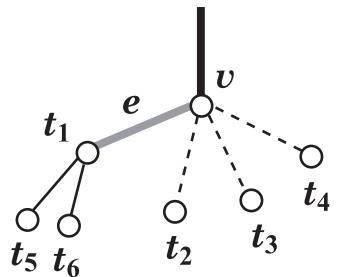

(a) force $\left(v t_{1}\right)$ in c-3

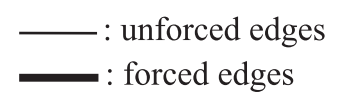

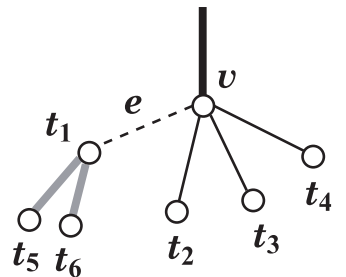

(b) delete $\left(v t_{1}\right)$ in c-3

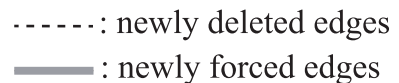

Fig. 5 Illustration of branching rule c-3, where vertex $v \in V_{\mathrm{f} 5}$ and vertex $t_{1} \in N_{U}\left(v ; V_{\mathrm{u} 3}\right)$.

vertex $t_{2}$ will be $w_{4}^{\prime}$ (resp., $\Delta_{4}, w_{5}^{\prime}$, and $\Delta_{5}$ ). Thus, the total weight decrease in the branch of delete $\left(v t_{1}\right)$ is at least $w_{5}^{\prime}-w_{4}^{\prime}+w_{3}^{\prime}+\beta_{4}$.

As a result, for the ordered pair $\left(\alpha_{4}, \beta_{4}\right)$ taking values in $\left\{\left(w_{4}^{\prime}, w_{4}^{\prime}\right),\left(w_{4}, \Delta_{4}\right),\left(\Delta_{5,3}^{\prime}, w_{5}^{\prime}\right),\left(\Delta_{5,3}, \Delta_{5}\right)\right\}$, we get the following four branching vectors:

$$
\left(w_{5}^{\prime}+w_{3}^{\prime}+2 m_{1}+\alpha_{4}, w_{5}^{\prime}-w_{4}^{\prime}+w_{3}^{\prime}+\beta_{4}\right) .
$$

Case c-3. Cases c-1 and c-2 are not applicable, and there exist vertices $v \in V_{\mathrm{f} 5}$ and $t_{1} \in N_{U}\left(v ; V_{\mathrm{u} 3}\right)$ (see Fig. 5): We branch on edge $v t_{1}$. Note that $N_{U}\left(t_{1}\right) \backslash\{v\}=\left\{t_{5}, t_{6}\right\}$.

In the branch of force $\left(v t_{1}\right)$, the edge $v t_{1}$ will be added to $F^{\prime}$ by the branching operation, and edges $v t_{2}, v t_{3}$ and $v t_{4}$ will be deleted from $G^{\prime}$ by the reduction rules. So, the weights of vertices $v$ and $t_{1}$ decrease by $w_{5}^{\prime}$ and $\Delta_{3}$, respectively. None of the vertices $t_{2}, t_{3}$ and $t_{4}$ can be an $\mathrm{f} 3$-vertex because it would have been chosen as an optimal edge in some previous case. Hence, each of the vertices $t_{2}, t_{3}$ and $t_{4}$ must be one of types $\mathrm{u} 3, \mathrm{f} 4, \mathrm{u} 4$, f5, and a u5-vertex, and each of their weights decreases by at least $m_{2}=\min \left\{w_{3}, \Delta_{4,3}^{\prime}, \Delta_{4,3}, \Delta_{5,4}^{\prime}, \Delta_{5,4}\right\}$. Thus, the total weight decrease in the branch of force $\left(v t_{1}\right)$ is at least $w_{5}^{\prime}+w_{3}-w_{3}^{\prime}+3 m_{2}$.

In the branch of delete $\left(v t_{1}\right)$, the edge $v t_{1}$ will be deleted from $G^{\prime}$ by the branching operation and edges $t_{1} t_{5}$ and $t_{1} t_{6}$ will be added to $F^{\prime}$ by the reduction rules. So, the weight of vertex $v$ decreases by $\Delta_{5,4}^{\prime}$ and the weight of vertex $t_{1}$ decreases by $w_{3}$. Each of the vertices $t_{5}$ and $t_{6}$ must be one of types $\mathrm{f} 3, \mathrm{u} 3, \mathrm{f} 4, \mathrm{u} 4, \mathrm{f} 5$, and a u5-vertex, and each of their weights decreases by at least $m_{3}=\min \left\{w_{3}^{\prime}, \Delta_{3}, w_{4}^{\prime}, \Delta_{4}, w_{5}^{\prime}, \Delta_{5}\right\}$. Thus, the total weight decrease in the branch of delete $\left(v t_{1}\right)$ is at least $w_{5}^{\prime}-w_{4}^{\prime}+w_{3}+2 m_{3}$.

As a result, we get the following branching vector:

$$
\left(w_{5}^{\prime}+w_{3}-w_{3}^{\prime}+3 m_{2}, w_{5}^{\prime}-w_{4}^{\prime}+w_{3}+2 m_{3}\right) .
$$

Case c-4. None of the previous cases are applicable, and there exist vertices $v \in V_{\mathrm{f} 5}$ and $t_{1} \in N_{U}\left(v ; V_{\mathrm{f} 4}\right)$ such that $N_{U}(v) \cap N_{U}\left(t_{1}\right)=\emptyset$ (see Fig. 6): We branch on edge $v t_{1}$. Note that $N_{U}\left(t_{1}\right) \backslash\{v\}=\left\{t_{5}, t_{6}\right\}$.

In the branch of force $\left(v t_{1}\right)$, the edge $v t_{1}$ will be added to $F^{\prime}$ by the branching operation, and edges $v t_{2}, v t_{3}, v t_{4}, t_{1} t_{5}$ and $t_{1} t_{6}$ will be deleted from $G^{\prime}$ by the reduction rules. So, the weight of vertex $v$ decreases by $w_{5}^{\prime}$ and the weight of vertex $t_{1}$ decreases by $w_{4}^{\prime}$. Each of the vertices $t_{2}, t_{3}$ and $t_{4}$ must be one of types $\mathrm{f} 4, \mathrm{u} 4, \mathrm{f} 5$, and a u5-vertex, and each of their weights decreases by at least $m_{4}=\min \left\{\Delta_{4,3}^{\prime}, \Delta_{4,3}, \Delta_{5,4}^{\prime}, \Delta_{5,4}\right\}$. Each of the vertices $t_{5}$ and $t_{6}$ must be one of types $\mathrm{f} 3, \mathrm{u} 3, \mathrm{f} 4, \mathrm{u} 4, \mathrm{f} 5$, 


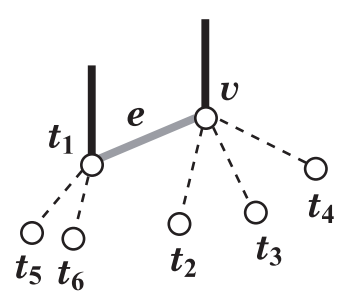

(a) force $\left(v t_{1}\right)$ in c-4

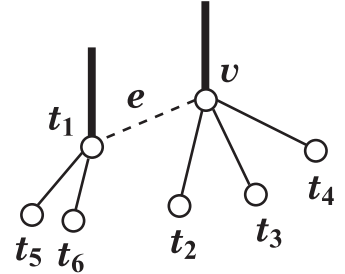

(b) delete $\left(v t_{1}\right)$ in c-4 . : newly deleted edges : newly forced edges unforced edges : forced edges

Fig. 6 Illustration of branching rule c-4, where vertex $v \in V_{\mathrm{f} 5}$ and vertex $t_{1} \in N_{U}\left(v ; V_{\mathrm{f} 4}\right)$, such that $N_{U}(v) \cap N_{U}\left(t_{1}\right)=\emptyset$.

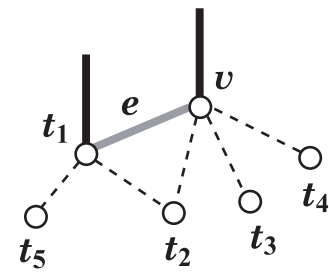

(a) force $\left(v t_{1}\right)$ in c-5(I)

: unforced edges forced edges

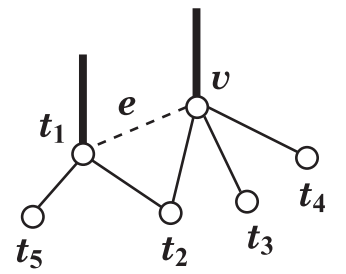

(b) delete $\left(v t_{1}\right)$ in c-5(I)

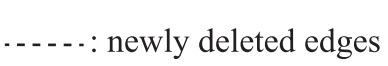
: newly forced edges
Fig. 7 Illustration of branching rule c-5(I), where vertex $v \in V_{\mathrm{f} 5}$ and vertex $t_{1} \in N_{U}\left(v ; V_{\mathrm{f} 4}\right)$, such that $N_{U}(v) \cap N_{U}\left(t_{1}\right)=\left\{t_{2}\right\}$.

and a u5-vertex, and each of their weights decreases by at least $m_{1}=\min \left\{w_{3}^{\prime}, w_{3}, \Delta_{4,3}^{\prime}, \Delta_{4,3}, \Delta_{5,4}^{\prime}, \Delta_{5,4}\right\}$. Thus, the total decrease in the branch of force $\left(v t_{1}\right)$ is at least $w_{5}^{\prime}+w_{4}^{\prime}+3 m_{4}+2 m_{1}$.

In the branch of delete $\left(v t_{1}\right)$, the edge $v t_{1}$ will be deleted from $G^{\prime}$ by the branching operation. So, the weight of vertex $v$ decreases by $\Delta_{5,4}^{\prime}$ and the weight of vertex $t_{1}$ decreases by $\Delta_{4,3}^{\prime}$. Thus, the total weight decrease in the branch of delete $\left(v t_{1}\right)$ is at least $w_{5}^{\prime}-w_{3}^{\prime}$.

As a result, we get the following branching vector:

$\left(w_{5}^{\prime}+w_{4}^{\prime}+3 m_{4}+2 m_{1}, w_{5}^{\prime}-w_{3}^{\prime}\right)$.

Case c-5. None of the previous cases are applicable, and there exist vertices $v \in V_{\mathrm{f} 5}$ and $t_{1} \in N_{U}\left(v ; V_{\mathrm{f} 4}\right)$ such that $N_{U}(v) \cap N_{U}\left(t_{1}\right) \neq \emptyset$. We distinguish two sub-cases, according to the cardinality of the intersection $N_{U}(v) \cap N_{U}\left(t_{1}\right)$,

c-5(I) $\left|N_{U}(v) \cap N_{U}\left(t_{1}\right)\right|=1$, and

c-5(II) $\left|N_{U}(v) \cap N_{U}\left(t_{1}\right)\right|=2$.

Case c-5(I). Without loss of generality, assume that $N_{U}(v) \cap$ $N_{U}\left(t_{1}\right)=\left\{t_{2}\right\}$ (see Fig. 7): We branch on edge $v t_{1}$. Note that $N_{U}\left(t_{1}\right) \backslash\{v\}=\left\{t_{5}\right\}$.

In the branch of force $\left(v t_{1}\right)$, the edge $v t_{1}$ will be added to $F^{\prime}$ by the branching operation, and edges $v t_{2}, v t_{3}, v t_{4}, t_{1} t_{2}$, and $t_{1} t_{5}$ will be deleted from $G^{\prime}$ by the reduction rules. So, the weight of vertex $v$ decreases by $w_{5}^{\prime}$ and the weight of vertex $t_{1}$ decreases by $w_{4}^{\prime}$. The vertex $t_{2}$ must be one of types $\mathrm{f} 4, \mathrm{u} 4, \mathrm{f} 5$, and a u5-vertex, and its weight decreases by at least $m_{5}=\min \left\{w_{4}^{\prime}, w_{4}, \Delta_{5,3}^{\prime}, \Delta_{5,3}\right\}$. Each of the vertices $t_{3}$ and $t_{4}$ must be one of types $\mathrm{f} 4, \mathrm{u} 4$, f5, and a u5-vertex, and each of their weights decreases by at least $m_{4}=\min \left\{\Delta_{4,3}^{\prime}, \Delta_{4,3}, \Delta_{5,4}^{\prime}, \Delta_{5,4}\right\}$. The vertex $t_{5}$ must be one of types f3, u3, f4, u4, f5, and a u5-vertex, and its weight decreases by at least $m_{1}=\min \left\{w_{3}^{\prime}, w_{3}, \Delta_{4,3}^{\prime}, \Delta_{4,3}, \Delta_{5,4}^{\prime}, \Delta_{5,4}\right\}$. Thus, the total weight decrease in the branch of force $\left(v t_{1}\right)$ is at least

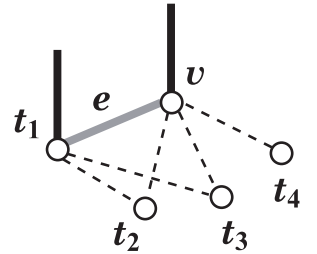

(a) force $\left(v t_{1}\right)$ in c-5(II)

: unforced edges : forced edges

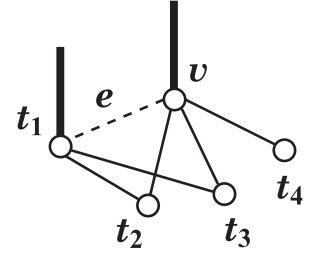

(b) $\operatorname{delete}\left(v t_{1}\right)$ in c-5(II)

: newly deleted edges : newly forced edges
Fig. 8 Illustration of branching rule c-5(II), where vertex $v \in V_{\mathrm{f} 5}$ and vertex $t_{1} \in N_{U}\left(v ; V_{\mathrm{f} 4}\right)$ such that $N_{U}(v) \cap N_{U}\left(t_{1}\right)=\left\{t_{2}, t_{3}\right\}$.

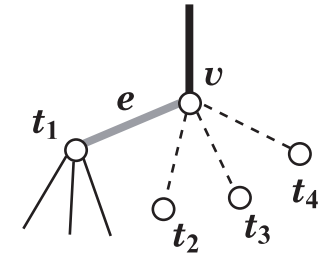

(a) force $\left(v t_{1}\right)$ in c-6

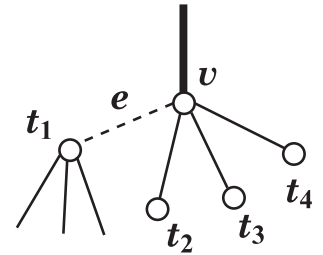

(b) delete $\left(v t_{1}\right)$ in c-6

\section{— : unforced edges : forced edges}

Fig. 9 Illustration of branching rule c-6, where vertex $v \in V_{\mathrm{f} 5}$ and vertex $t_{1} \in N_{U}\left(v ; V_{\mathrm{u} 4}\right)$.

$w_{5}^{\prime}+w_{4}^{\prime}+m_{5}+2 m_{4}+m_{1}$.

In the branch of delete $\left(v t_{1}\right)$, the edge $v t_{1}$ will be deleted from $G^{\prime}$ by the branching operation. So, the weight of vertex $v$ decreases by $\Delta_{5,4}^{\prime}$, and the weight of vertex $t_{1}$ decreases by $\Delta_{4,3}^{\prime}$. Thus, the total weight decrease in the branch of $\operatorname{delete}\left(v t_{1}\right)$ is at least $w_{5}^{\prime}-w_{3}^{\prime}$.

As a result, we get the following branching vector:

$$
\left(w_{5}^{\prime}+w_{4}^{\prime}+m_{5}+2 m_{4}+m_{1}, w_{5}^{\prime}-w_{3}^{\prime}\right)
$$

Case c-5(II). Without loss of generality, assume that $N_{U}(v) \cap$ $N_{U}\left(t_{1}\right)=\left\{t_{2}, t_{3}\right\}$ (see Fig. 8): We branch on edge $v t_{1}$.

In the branch of force $\left(v t_{1}\right)$, the edge $v t_{1}$ will be added to $F^{\prime}$ by the branching operation, and edges $v t_{2}, v t_{3}, v t_{4}, t_{1} t_{2}$ and $t_{1} t_{3}$ will be deleted from $G^{\prime}$ by the reduction rules. So, the weight of vertex $v$ decreases by $w_{5}^{\prime}$ and the weight of vertex $t_{1}$ decreases by $w_{4}^{\prime}$. Each of the vertices $t_{2}$ and $t_{3}$ must be one of types $\mathrm{f} 4, \mathrm{u} 4, \mathrm{f} 5$, and a u5-vertex, and each of their weights decreases by at least $m_{5}=\min \left\{w_{4}^{\prime}, w_{4}, \Delta_{5,3}^{\prime}, \Delta_{5,3}\right\}$. The vertex $t_{4}$ must be one of types $\mathrm{f} 4, \mathrm{u} 4$, f5, and a u5-vertex, and its weight decreases by at least $m_{4}=\min \left\{\Delta_{4,3}^{\prime}, \Delta_{4,3}, \Delta_{5,4}^{\prime}, \Delta_{5,4}\right\}$. Thus, the total weight decrease in the branch of force $\left(v t_{1}\right)$ is at least $w_{5}^{\prime}+w_{4}^{\prime}+2 m_{5}+m_{4}$.

In the branch of delete $\left(v t_{1}\right)$, the edge $v t_{1}$ will be deleted from $G^{\prime}$ by the branching operation. So, the weight of vertex $v$ decreases by $\Delta_{5,4}^{\prime}$, and the weight of vertex $t_{1}$ decreases by $\Delta_{4,3}^{\prime}$. Thus, the total weight decrease in the branch of delete $\left(v t_{1}\right)$ is at least $w_{5}^{\prime}-w_{3}^{\prime}$.

As a result, we get the following branching vector:

$$
\left(w_{5}^{\prime}+w_{4}^{\prime}+2 m_{5}+m_{4}, w_{5}^{\prime}-w_{3}^{\prime}\right) .
$$

Case c-6. None of the previous cases are applicable, and there exist vertices $v \in V_{\mathrm{f} 5}$ and $t_{1} \in N_{U}\left(v ; V_{\mathrm{u} 4}\right)$ (see Fig. 9): We branch on edge $v t_{1}$. 


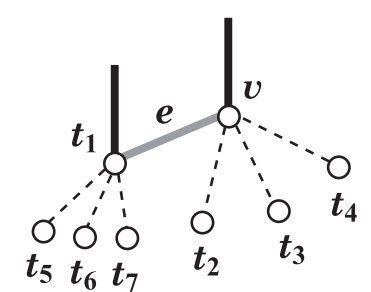

(a) force $\left(v t_{1}\right)$ in c-7

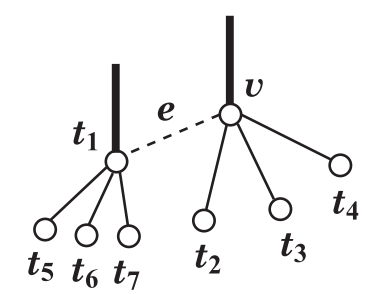

(b) $\operatorname{delete}\left(v t_{1}\right)$ in c-7

: newly deleted edges : newly forced edges
Fig. 10 Illustration of branching rule c-7, where vertex $v \in V_{\mathrm{f} 5}$ and vertex $t_{1} \in N_{U}\left(v ; V_{\mathrm{f} 5}\right)$, such that $N_{U}(v) \cap N_{U}\left(t_{1}\right)=\emptyset$.

In the branch of force $\left(v t_{1}\right)$, the edge $v t_{1}$ will be added to $F^{\prime}$ by the branching operation, and edges $v t_{2}, v t_{3}$ and $v t_{4}$ will be deleted from $G^{\prime}$ by the reduction rules. So, the weight of vertex $v$ decreases by $w_{5}^{\prime}$ and the weight of vertex $t_{1}$ decreases by $\Delta_{4}$. Each of the vertices $t_{2}, t_{3}$ and $t_{4}$ must be one of types $\mathrm{u} 4, \mathrm{f} 5$, and a u5-vertex, and each of their weights decreases by at least $m_{6}=\min \left\{\Delta_{4,3}, \Delta_{5,4}^{\prime}, \Delta_{5,4}\right\}$. Thus, the total weight decrease in the branch of force $\left(v t_{1}\right)$ is at least $w_{5}^{\prime}+w_{4}-w_{4}^{\prime}+3 m_{6}$.

In the branch of delete $\left(v t_{1}\right)$, the edge $v t_{1}$ will be deleted from $G^{\prime}$ by the branching operation. So, the weight of vertex $v$ decreases by $\Delta_{5,4}^{\prime}$ and the weight of vertex $t_{1}$ decreases by $\Delta_{4,3}$. Thus, the total weight decrease in the branch of $\operatorname{delete}\left(v t_{1}\right)$ is at least $w_{5}^{\prime}-w_{4}^{\prime}+w_{4}-w_{3}$.

As a result, we get the following branching vector:

$$
\left(w_{5}^{\prime}+w_{4}-w_{4}^{\prime}+3 m_{6}, w_{5}^{\prime}-w_{4}^{\prime}+w_{4}-w_{3}\right)
$$

Case c-7. None of the previous cases are applicable, and there exist vertices $v \in V_{\mathrm{f} 5}$ and $t_{1} \in N_{U}\left(v ; V_{\mathrm{f} 5}\right)$ such that $N_{U}(v) \cap N_{U}\left(t_{1}\right)=\emptyset$ (see Fig. 10): We branch on edge $v t_{1}$. Note that $N_{U}\left(t_{1}\right) \backslash\{v\}=\left\{t_{5}, t_{6}, t_{7}\right\}$.

In the branch of force $\left(v t_{1}\right)$, the edge $v t_{1}$ will be added to $F^{\prime}$ by the branching operation, and edges $v t_{2}, v t_{3}, v t_{4}, t_{1} t_{5}, t_{1} t_{6}$ and $t_{1} t_{7}$ will be deleted from $G^{\prime}$ by the reduction rules. So, both weights of vertex $v$ and vertex $t_{1}$ each decrease by $w_{5}^{\prime}$. Each of the vertices $t_{2}, t_{3}, t_{4}, t_{5}, t_{6}$ and $t_{7}$ must be a type f5, or a u5-vertex, and each of their weights decreases by at least $m_{7}=\min \left\{\Delta_{5,4}^{\prime}, \Delta_{5,4}\right\}$. Thus, the total weight decrease in the branch of force $\left(v t_{1}\right)$ is at least $2 w_{5}^{\prime}+6 m_{7}$.

In the branch of delete $\left(v t_{1}\right)$, the edge $v t_{1}$ will be deleted from $G^{\prime}$ by the branching operation. So, both weights of vertices $v$ and $t_{1}$ each decrease by $\Delta_{5,4}^{\prime}$. Thus, the total weight decrease in the branch of delete $\left(v t_{1}\right)$ is at least $2 w_{5}^{\prime}-2 w_{4}^{\prime}$.

As a result, we get the following branching vector:

$$
\left(2 w_{5}^{\prime}+6 m_{7}, 2 w_{5}^{\prime}-2 w_{4}^{\prime}\right)
$$

Case c-8. None of the previous cases are applicable, and there exist vertices $v \in V_{\mathrm{f} 5}$ and $t_{1} \in N_{U}\left(v ; V_{\mathrm{f} 5}\right)$ such that $N_{U}(v) \cap N_{U}\left(t_{1}\right) \neq \emptyset$. We distinguish three sub-cases, according to the cardinality of the intersection $N_{U}(v) \cap N_{U}\left(t_{1}\right)$,
c-8(I) $\quad\left|N_{U}(v) \cap N_{U}\left(t_{1}\right)\right|=1$,
c-8(II) $\quad\left|N_{U}(v) \cap N_{U}\left(t_{1}\right)\right|=2$, and
c-8(III) $\left|N_{U}(v) \cap N_{U}\left(t_{1}\right)\right|=3$.

Case c-8(I). Without loss of generality, assume that $N_{U}(v) \cap$

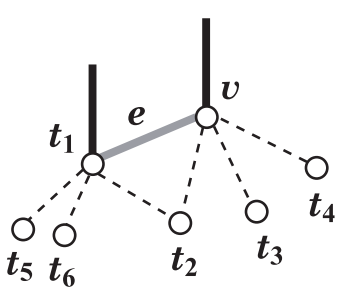

(a) force $\left(v t_{1}\right)$ in c-8(I)

: unforced edges

: forced edges

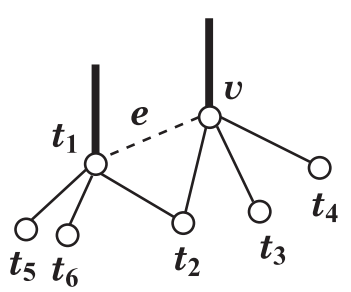

(b) $\operatorname{delete}\left(v t_{1}\right)$ in $\mathrm{c}-8(\mathrm{I})$ : newly deleted edges : newly forced edges
Fig. 11 Illustration of branching rule c-8(I), where vertex $v \in V_{\mathrm{f} 5}$ and vertex $t_{1} \in N_{U}\left(v ; V_{\mathrm{f} 5}\right)$, such that $N_{U}(v) \cap N_{U}\left(t_{1}\right)=\left\{t_{2}\right\}$.

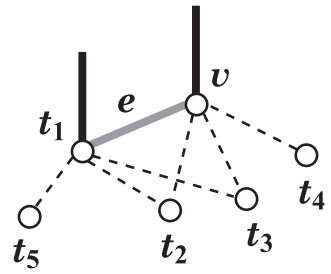

(a) force $\left(v t_{1}\right)$ in c-8(II)

: unforced edges
- forced edges $\quad$ : newly forced edges

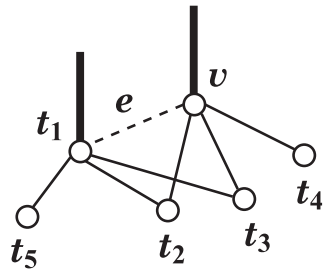

(b) delete $\left(v t_{1}\right)$ in c-8(II)
Fig. 12 Illustration of branching rule c-8(II), where vertex $v \in V_{\mathrm{f} 5}$ and vertex $t_{1} \in N_{U}\left(v ; V_{\mathrm{f} 5}\right)$, such that $N_{U}(v) \cap N_{U}\left(t_{1}\right)=\left\{t_{2}, t_{3}\right\}$.

$N_{U}\left(t_{1}\right)=\left\{t_{2}\right\}$ (see Fig. 11): We branch on edge $v t_{1}$. Note that $N_{U}\left(t_{1}\right) \backslash\{v\}=\left\{t_{5}, t_{6}\right\}$.

In the branch of force $\left(v t_{1}\right)$, the edge $v t_{1}$ will be added to $F^{\prime}$ by the branching operation, and edges $v t_{2}, v t_{3}, v t_{4}, t_{1} t_{2}, t_{1} t_{5}$ and $t_{1} t_{6}$ will be deleted from $G^{\prime}$ by the reduction rules. So, both weights of vertex $v$ and vertex $t_{1}$ each decrease by $w_{5}^{\prime}$. The vertex $t_{2}$ must be a type f5 or a u5-vertex, and its weight decreases by at least $m_{8}=\min \left\{\Delta_{5,3}^{\prime}, \Delta_{5,3}\right\}$. Each of the vertices $t_{3}, t_{4}, t_{5}$ and $t_{6}$ must be a type $\mathrm{f} 5$, or a u5-vertex, and each of their weights decreases by at least $m_{7}=\min \left\{\Delta_{5,4}^{\prime}, \Delta_{5,4}\right\}$. Thus, the total weight decrease in the branch of force $\left(v t_{1}\right)$ is at least $2 w_{5}^{\prime}+4 m_{7}+m_{8}$.

In the branch of delete $\left(v t_{1}\right)$, the edge $v t_{1}$ will be deleted from $G^{\prime}$ by the branching operation. So, both weights of vertices $v$ and $t_{1}$ each decreases by $\Delta_{5,4}^{\prime}$. Thus, the total weight decrease in the branch of delete $\left(v t_{1}\right)$ is at least $2 w_{5}^{\prime}-2 w_{4}^{\prime}$.

As a result, we get the following branching vector:

$$
\left(2 w_{5}^{\prime}+4 m_{7}+m_{8}, 2 w_{5}^{\prime}-2 w_{4}^{\prime}\right)
$$

Case c-8(II). Without loss of generality, assume that $N_{U}(v) \cap$ $N_{U}\left(t_{1}\right)=\left\{t_{2}, t_{3}\right\}$ (see Fig. 12): We branch on edge $v t_{1}$. Note that $N_{U}\left(t_{1}\right) \backslash\{v\}=\left\{t_{5}\right\}$.

In the branch of force $\left(v t_{1}\right)$, the edge $v t_{1}$ will be added to $F^{\prime}$ by the branching operation, and edges $v t_{2}, v t_{3}, v t_{4}, t_{1} t_{2}, t_{1} t_{3}$ and $t_{1} t_{5}$ will be deleted from $G^{\prime}$ by the reduction rules. So, both weights of vertex $v$ and vertex $t_{1}$ each decrease by $w_{5}^{\prime}$. Each of the vertices $t_{2}$ and $t_{3}$ must be a type f5, or a u5-vertex, and each of their weights decreases by at least $m_{8}=\min \left\{\Delta_{5,3}^{\prime}, \Delta_{5,3}\right\}$. Each of the vertices $t_{4}$ and $t_{5}$ must be a type f5, or a u5-vertex, and each of their weights decreases by at least $m_{7}=\min \left\{\Delta_{5,4}^{\prime}, \Delta_{5,4}\right\}$. Thus, the total weight decrease in the branch of force $\left(v t_{1}\right)$ is at least $2 w_{5}^{\prime}+2 m_{8}+2 m_{7}$. 


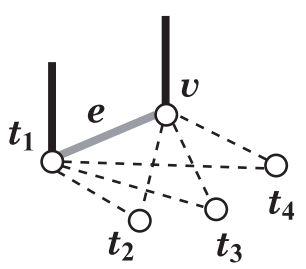

(a) force $\left(v t_{1}\right)$ in c-8(III)

: unforced edges forced edges

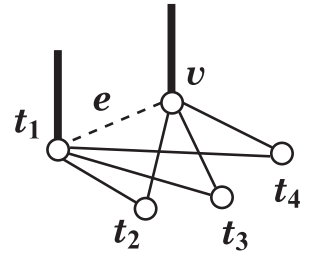

(b) delete $\left(v t_{1}\right)$ in c-8(III)

. . . . : newly deleted edges newly forced edges
Fig. 13 Illustration of branching rule c-8(III), where vertex $v \in V_{\text {f5 }}$ and vertex $t_{1} \in N_{U}\left(v ; V_{\mathrm{f} 5}\right)$, such that $N_{U}(v) \cap N_{U}\left(t_{1}\right)=\left\{t_{2}, t_{3}, t_{4}\right\}$.

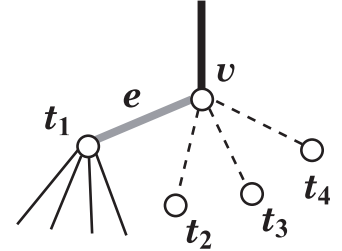

(a) force $\left(v t_{1}\right)$ in c-9

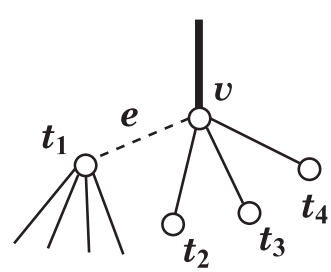

(b) $\operatorname{delete}\left(v t_{1}\right)$ in c-9
: unforced edges forced edges

...... newly deleted edges : newly forced edges

Fig. 14 Illustration of branching rule c-9, where vertex $v \in V_{\mathrm{f} 5}$ and vertex $t_{1} \in N_{U}\left(v ; V_{\mathrm{u} 5}\right)$.

In the branch of delete $\left(v t_{1}\right)$, the edge $v t_{1}$ will be deleted from $G^{\prime}$ by the branching operation. So, both weights of vertex $v$ and vertex $t_{1}$ each decreases by $\Delta_{5,4}^{\prime}$. Thus, the total weight decrease in the branch of delete $\left(v t_{1}\right)$ is at least $2 w_{5}^{\prime}-2 w_{4}^{\prime}$.

As a result, we get the following branching vector:

$$
\left(2 w_{5}^{\prime}+2 m_{8}+2 m_{7}, 2 w_{5}^{\prime}-2 w_{4}^{\prime}\right) .
$$

Case c-8(III). We have that $N_{U}(v) \cap N_{U}\left(t_{1}\right)=\left\{t_{2}, t_{3}, t_{4}\right\}$ (see Fig. 13): We branch on edge $v t_{1}$.

In the branch of force $\left(v t_{1}\right)$, the edge $v t_{1}$ will be added to $F^{\prime}$ by the branching operation, and edges $v t_{2}, v t_{3}, v t_{4}, t_{1} t_{2}, t_{1} t_{3}$ and $t_{1} t_{4}$ will be deleted from $G^{\prime}$ by the reduction rules. So, both weights of vertex $v$ and vertex $t_{1}$ each decrease by $w_{5}^{\prime}$. Each of the vertices $t_{2}, t_{3}$, and $t_{4}$ must be a type f5, or a u5-vertex, and each of their weights decreases by at least $m_{8}=\min \left\{\Delta_{5,3}^{\prime}, \Delta_{5,3}\right\}$. Thus, the total weight decrease in the branch of force $\left(v t_{1}\right)$ is at least $2 w_{5}^{\prime}+3 m_{8}$.

In the branch of delete $\left(v t_{1}\right)$, the edge $v t_{1}$ will be deleted from $G^{\prime}$ by the branching operation. So, both weights of vertex $v$ and vertex $t_{1}$ each decrease by $\Delta_{5,4}^{\prime}$. Thus, the total weight decrease in the branch of $\operatorname{delete}\left(v t_{1}\right)$ is at least $2 w_{5}^{\prime}-2 w_{4}^{\prime}$.

As a result, we get the following branching vector:

$$
\left(2 w_{5}^{\prime}+3 m_{8}, 2 w_{5}^{\prime}-2 w_{4}^{\prime}\right)
$$

Case c-9. None of the previous cases are applicable, and there exist vertices $v \in V_{\mathrm{f} 5}$ and $t_{1} \in N_{U}\left(v ; V_{\mathrm{u} 5}\right)$ (see Fig. 14): We branch on edge $v t_{1}$.

In the branch of force $\left(v t_{1}\right)$, the edge $v t_{1}$ will be added to $F^{\prime}$ by the branching operation, and edges $v t_{2}, v t_{3}$ and $v t_{4}$ will be deleted from $G^{\prime}$ by the reduction rules. So, the weight of vertex $v$ decreases by $w_{5}^{\prime}$, and the weight of vertex $t_{1}$ decreases by $\Delta_{5}$. Each of the vertices $t_{2}, t_{3}$ and $t_{4}$ must be a type u5-vertex, and each of their weights decrease by $\Delta_{5,4}$. Thus, the total weight decrease in

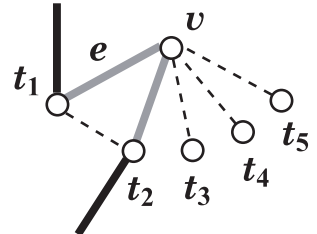

(a) force $\left(v t_{1}\right)$ in c-10(I)

— : unforced edges : forced edges

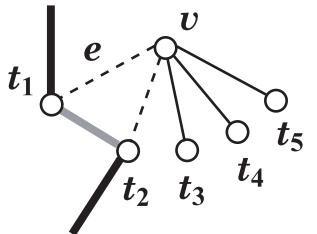

(b) $\operatorname{delete}\left(v t_{1}\right)$ in c-10(I)

.... : newly deleted edges : newly forced edges
Fig. 15 Illustration for c-10(I) where vertices $v \in V_{\mathrm{u} 5}$ and $t_{1} \in N_{U}\left(v ; V_{\mathrm{f} 3}\right)$, such that $N_{U}(v) \cap N_{U}\left(t_{1}\right)=\left\{t_{2}\right\}, t_{2}$ is an $\mathrm{f} 3$-vertex.

the branch of force $\left(v t_{1}\right)$ is at least $4 w_{5}-3 w_{4}$.

In the branch of delete $\left(v t_{1}\right)$, the edge $v t_{1}$ will be deleted from $G^{\prime}$ by the branching operation. Thus, the weight of vertex $v$ decreases by $\Delta_{5,4}^{\prime}$, and the weight of vertex $t_{1}$ decreases by $\Delta_{5,4}$. The total weight decrease in the branch of $\operatorname{delete}\left(v t_{1}\right)$ is at least $w_{5}+w_{5}^{\prime}-w_{4}-w_{4}^{\prime}$.

Then, we get the following branching vector:

$$
\left(4 w_{5}-3 w_{4}, w_{5}+w_{5}^{\prime}-w_{4}-w_{4}^{\prime}\right)
$$

\subsection{Branching on Edges Around u5-vertices (Branching Rules c-10 to c-15)}

If none of the first nine conditions can be executed, this means that the instance of forced TSP has no f5-vertices. But this does not mean that the maximum degree of the reduced instance at this point is at most 4 , since there might still be u5-vertices. This section derives branching vectors for branchings on an optimal edge $e=v t_{1}$ incident to a 45 -vertex $v$, distinguishing the conditions of six cases, c-10 to c- 15 .

When branching on an edge $v t_{1}$ incident to a u5-vertex $v$, let $a$ and $b$ be lower bound on the decrease in measure due to the force $(e)$ and $\operatorname{delete}(e)$ operations, respectively. In the branch of force $\left(v t_{1}\right)$, the vertex $v$ will become an f5-vertex. Therefore, at this point, some of the branching rules of cases c- 1 to c- 9 become immediately applicable. Let $(A, B)$ be the branching vectors of the branching rules that become immediately applicable. Then we regard the overall branching vectors to be $(a+A, a+B, b)$ [7].

Case c-10. There are no more f5-vertices, and there exist vertices $v \in V_{\mathrm{u} 5}$ and $t_{1} \in N_{U}\left(v ; V_{\mathrm{f} 3}\right)$ such that $N_{U}(v) \cap N_{U}\left(t_{1}\right) \neq \emptyset$. Note that it holds $\left|N_{U}(v) \cap N_{U}\left(t_{1}\right)\right|=1$. Without loss of generality, assume that $N_{U}(v) \cap N_{U}\left(t_{1}\right)=\left\{t_{2}\right\}$. We distinguish five sub-cases, according to the type of vertex $t_{2}$, the unique vertex in the intersection $N_{U}(v) \cap N_{U}\left(t_{1}\right)$,

c-10(I) $\quad t_{2}$ is an $\mathrm{f} 3$-vertex,

c-10(II) $\quad t_{2}$ is a u3-vertex,

c-10(III) $t_{2}$ is an f4-vertex,

c-10(IV) $t_{2}$ is a u4-vertex, and

c-10(V) $\quad t_{2}$ is a u5-vertex.

Case c-10(I). Vertex $t_{2}$ is an f3-vertex (see Fig. 15): We branch on edge $v t_{1}$.

In the branch of force $\left(v t_{1}\right)$, the edge $v t_{1}$ will be added to $F^{\prime}$ by the branching operation, edges $v t_{3}, v t_{4}, v t_{5}$ and $t_{1} t_{2}$ will be deleted from $G^{\prime}$ by the reduction rules, and the edge $v t_{2}$ will be added to $F^{\prime}$ by the reduction rules. Hence, the weight of vertex $v$ decreases by $w_{5}$, and the weight of each of the vertices $t_{1}$ and $t_{2}$ 


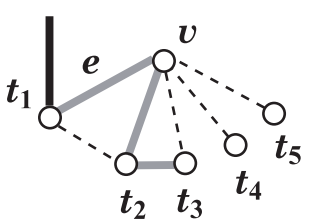

(a) force $\left(v t_{1}\right)$ in c-10(II)

: unforced edges forced edges

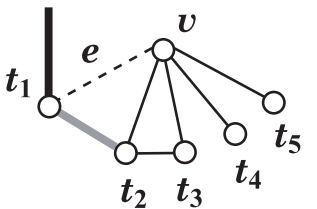

(b) delete $\left(v t_{1}\right)$ in c-10(II)

-...-. : newly deleted edges : newly forced edges
Fig. 16 Illustration for c-10(II) where vertices $v \in V_{\mathrm{u} 5}$ and $t_{1} \in N_{U}\left(v ; V_{\mathrm{f} 3}\right)$, such that $N_{U}(v) \cap N_{U}\left(t_{1}\right)=\left\{t_{2}\right\}, t_{2}$ is a u3-vertex, and the unique neighbor of $t_{2}$ not in $\left\{t_{1}, v\right\}$ is in $N_{U}(v)$.

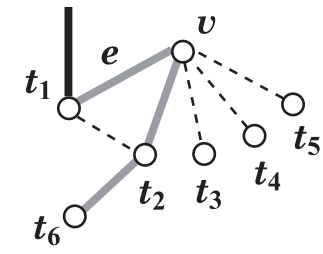

(a) force $\left(v t_{1}\right)$ in c-10(II)

: unforced edges forced edges

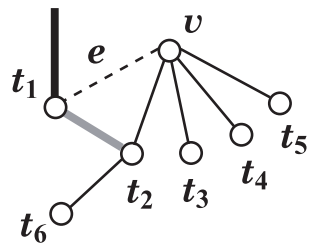

(b) $\operatorname{delete}\left(v t_{1}\right)$ in c-10(II)

..... : newly deleted edges : newly forced edges
Fig. 17 Illustration for c-10(II) where vertices $v \in V_{\mathrm{u} 5}$ and $t_{1} \in N_{U}\left(v ; V_{\mathrm{f} 3}\right)$, such that $N_{U}(v) \cap N_{U}\left(t_{1}\right)=\left\{t_{2}\right\}, t_{2}$ is a u3-vertex, and the unique neighbor of $t_{2}$ not in $\left\{t_{1}, v\right\}$ is not in $N_{U}(v)$.

decrease by $w_{3}^{\prime}$. Each of the vertices $t_{3}, t_{4}$ and $t_{5}$ must be one of types f3, u3, f4, u4, and a u5-vertex, and each of their weights decreases by at least $m_{10}=\min \left\{w_{3}^{\prime}, w_{3}, \Delta_{4,3}^{\prime}, \Delta_{4,3}, \Delta_{5,4}\right\}$. Thus, the total weight decrease in the branch of force $\left(v t_{1}\right)$ is at least $\left(w_{5}+2 w_{3}^{\prime}+3 m_{10}\right)$.

In the branch of delete $\left(v t_{1}\right)$, the edge $v t_{1}$ will be deleted from $G^{\prime}$ by the branching operation, the edge $v t_{2}$ will be deleted from $G^{\prime}$ by the reduction rules, and the edge $t_{1} t_{2}$ will be added to $F^{\prime}$ by the reduction rules. Hence, the weight of vertex $v$ decreases by $\Delta_{5,3}$, and both weights of vertices $t_{1}$ and $t_{2}$ each decrease by $w_{3}^{\prime}$. Thus, the total weight decrease in the branch of $\operatorname{delete}\left(v t_{1}\right)$ is at least $w_{5}-w_{3}+2 w_{3}^{\prime}$.

As a result, we get the following branching vector:

$$
\left(w_{5}+2 w_{3}^{\prime}+3 m_{10}, w_{5}-w_{3}+2 w_{3}^{\prime}\right) .
$$

Case c-10(II). Vertex $t_{2}$ is a u3-vertex (see Figs. 16 and 17): We branch on edge $v t_{1}$. Note that $N_{U}\left(t_{2}\right) \backslash\left\{v, t_{1}\right\}=\left\{t_{6}\right\}$.

In the branch of delete $\left(v t_{1}\right)$, the edge $v t_{1}$ will be deleted from $G^{\prime}$ by the branching operation, and the edge $t_{1} t_{2}$ will be added to $F^{\prime}$ by the reduction rules. Hence, the weight of vertex $v$ decreases by $\Delta_{5,4}$, and the weight of vertex $t_{1}$ decreases by $w_{3}^{\prime}$, and the weight of vertex $t_{2}$ decreases by $\Delta_{3}$. Thus, the total weight decrease in the branch of delete $\left(v t_{1}\right)$ is at least $w_{5}-w_{4}+w_{3}^{\prime}+w_{3}-w_{3}^{\prime}$.

In the branch of force $\left(v t_{1}\right)$, the edge $v t_{1}$ will be added to $F^{\prime}$ by the branching operation, the edge $v t_{2}$ will be added to $F^{\prime}$ by the reduction rules, and edges $v t_{3}, v t_{4}, v t_{5}$ and $t_{1} t_{2}$ will be deleted from $G^{\prime}$ by the reduction rules.

For vertex $t_{6}$ it holds either a) $t_{6} \in N_{U}(v)$ (see Fig. 16), or b) $t_{6} \notin N_{U}(v)$ (see Fig. 17).

If the vertex $t_{6} \in N_{U}(v) \cap N_{U}\left(t_{2}\right)$, then without loss of generality we assume that $t_{6}$ is $t_{3}$ (see Fig. 16). Then, the edge $t_{2} t_{3}$ will be added to $F^{\prime}$ by the reduction rules. Hence, the weight of

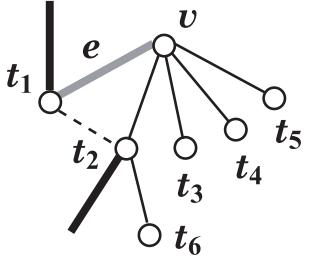

(a) force $\left(v t_{1}\right)$ in c-10(III)

: unforced edges forced edges

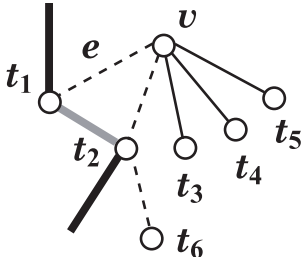

(b) delete $\left(v t_{1}\right)$ in c-10(III)

-... : newly deleted edges : newly forced edges
Fig. 18 Illustration for c-10(III) where vertices $v \in V_{\mathrm{u} 5}$ and $t_{1} \in N_{U}\left(v ; V_{\mathrm{f} 3}\right)$, such that $N_{U}(v) \cap N_{U}\left(t_{1}\right)=\left\{t_{2}\right\}, t_{2}$ is an f4-vertex, and $N_{U}(v) \cap$ $N_{U}\left(t_{2}\right) \backslash\left\{t_{1}\right\}=\emptyset$.

vertex $v$ decreases by $w_{5}$, the weight of vertex $t_{1}$ decreases by $w_{3}^{\prime}$, and the weight of vertex $t_{2}$ decreases by $w_{3}$. The vertex $t_{3}$ must be one of types $\mathrm{f} 3, \mathrm{u} 3, \mathrm{f} 4, \mathrm{u} 4$, and a u5-vertex, and its weight decreases by at least $m_{11}=\min \left\{w_{3}^{\prime}, w_{3}, w_{4}^{\prime}, w_{4}-w_{3}^{\prime}, w_{5}-w_{4}^{\prime}\right\}$. Each of the vertices $t_{4}$ and $t_{5}$ must be one of types $\mathrm{f} 3, \mathrm{u} 3, \mathrm{f} 4, \mathrm{u} 4$, and a u5-vertex, and each of their weights decreases by at least $m_{10}=$ $\min \left\{w_{3}^{\prime}, w_{3}, \Delta_{4,3}^{\prime}, \Delta_{4,3}, \Delta_{5,4}\right\}$. Thus, the total weight decrease in the branch of force $\left(v t_{1}\right)$ is at least $w_{5}+w_{3}^{\prime}+w_{3}+m_{11}+2 m_{10}$.

As a result, we get the following branching vector:

$\left(w_{5}+w_{3}^{\prime}+w_{3}+m_{11}+2 m_{10}, w_{5}-w_{4}+w_{3}\right)$.

Next, if the vertex $t_{6} \notin N_{U}(v) \cap N_{U}\left(t_{2}\right)$ (see Fig. 17), then the edge $t_{2} t_{6}$ will be added to $F^{\prime}$ by the reduction rules. Hence, the weight of vertex $v$ decreases by $w_{5}$, the weight of vertex $t_{1}$ decreases by $w_{3}^{\prime}$, and the weight of vertex $t_{2}$ decreases by $w_{3}$. The vertex $t_{6}$ must be one of types $\mathrm{f} 3, \mathrm{u} 3, \mathrm{f} 4, \mathrm{u} 4$, and a u5-vertex, and its weight decreases by at least $m_{9}=\min \left\{w_{3}^{\prime}, \Delta_{3}, w_{4}^{\prime}, \Delta_{4}, \Delta_{5}\right\}$. Each of the vertices $t_{3}, t_{4}$ and $t_{5}$ must be one of types f3, u3, $\mathrm{f} 4, \mathrm{u} 4$, and a u5-vertex, and each of their weights decreases by at least $m_{10}=\min \left\{w_{3}^{\prime}, w_{3}, \Delta_{4,3}^{\prime}, \Delta_{4,3}, \Delta_{5,4}\right\}$. Thus, the total weight decrease in the branch of force $\left(v t_{1}\right)$ is at least $w_{5}+w_{3}+w_{3}^{\prime}+m_{9}+3 m_{10}$.

As a result, we get the following branching vector:

$\left(w_{5}+w_{3}+w_{3}^{\prime}+m_{9}+3 m_{10}, w_{5}-w_{4}+w_{3}\right)$.

Case c-10(III). Vertex $t_{2}$ is an f4-vertex (see Fig. 18): We branch on edge $v t_{1}$. Note that $N_{U}\left(t_{2}\right) \backslash\left\{v, t_{1}\right\}=\left\{t_{6}\right\}$.

In the branch of force $\left(v t_{1}\right)$, the edge $v t_{1}$ will be added to $F^{\prime}$ by the branching operation, and the edge $t_{1} t_{2}$ will be deleted from $G^{\prime}$ by the reduction rules. Hence, the weight of vertex $v$ decreases by $\Delta_{5}$, the weight of vertex $t_{1}$ decreases by $w_{3}^{\prime}$, and the weight of vertex $t_{2}$ decreases by $\Delta_{4,3}^{\prime}$. Thus, the total weight decrease in the branch of force $\left(v t_{1}\right)$ is at least $w_{5}-w_{5}^{\prime}+w_{3}^{\prime}+w_{4}^{\prime}-w_{3}^{\prime}$.

In the branch of delete $\left(v t_{1}\right)$, the edge $v t_{1}$ will be deleted from $G^{\prime}$ by the branching operation, the edge $t_{1} t_{2}$ will be added to $F^{\prime}$ by the reduction rules, and edges $v t_{2}$ and $t_{2} t_{6}$ will be deleted from $G^{\prime}$ by the reduction rules. Hence, the weight of vertex $v$ decreases by $\Delta_{5,3}$, the weight of vertex $t_{1}$ decreases by $w_{3}^{\prime}$, and the weight of vertex $t_{2}$ decreases by $w_{4}^{\prime}$. The vertex $t_{6}$ must be one of types $\mathrm{f} 3, \mathrm{u} 3, \mathrm{f} 4, \mathrm{u} 4$, and a u5-vertex, and its weight decreases by at least $m_{10}=\min \left\{w_{3}^{\prime}, w_{3}, \Delta_{4,3}^{\prime}, \Delta_{4,3}, \Delta_{5,4}\right\}$. Thus, the total weight decrease in the branch of delete $\left(v t_{1}\right)$ is at least $w_{5}-w_{3}+w_{3}^{\prime}+w_{4}^{\prime}+m_{10}$.

As a result, we get the following branching vector:

$\left(w_{5}-w_{5}^{\prime}+w_{4}^{\prime}, w_{5}-w_{3}+w_{3}^{\prime}+w_{4}^{\prime}+m_{10}\right)$. 


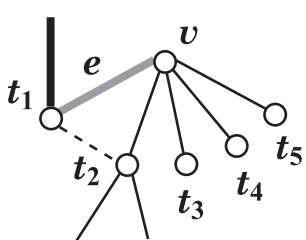

(a) force $\left(v t_{1}\right)$ in c-10(IV)

: unforced edges : forced edges

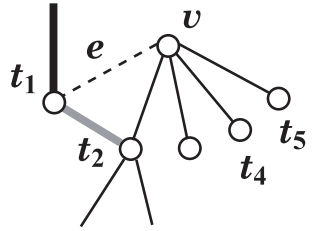

(b) delete $\left(v t_{1}\right)$ in c-10(IV)

- . - . . : newly deleted edges : newly forced edges
Fig. 19 Illustration for c-10(IV) where vertices $v \in V_{\mathrm{u} 5}$ and $t_{1} \in N_{U}\left(v ; V_{\mathrm{f} 3}\right)$, such that $N_{U}(v) \cap N_{U}\left(t_{1}\right)=\left\{t_{2}\right\}, t_{2}$ is a u4-vertex.

In the branch of force $\left(v t_{1}\right)$, the vertex $v$ will become an f5vertex, and at this point, one of the branching rules of cases c1 and c- 2 becomes immediately applicable, because an optimal edge for the next branching operation is at least as good as the edge $v t_{2}$. Therefore, for the case where the vertex $t_{3}$ is either an $\mathrm{f} 3$ or a $\mathrm{u} 3$-vertex, for each of the 19 branching vectors $(A, B)$ of cases c-1 and c-2, we get the following branching vector:

$$
\left(w_{5}-w_{5}^{\prime}+w_{4}^{\prime}+A, w_{5}-w_{5}^{\prime}+w_{4}^{\prime}+B, w_{5}-w_{3}+w_{3}^{\prime}+w_{4}^{\prime}+m_{10}\right) .
$$

Case c-10(IV). Vertex $t_{2}$ is a u4-vertex (see Fig. 19): We branch on edge $v t_{1}$.

In the branch of force $\left(v t_{1}\right)$, the edge $v t_{1}$ will be added to $F^{\prime}$ by the branching operation, and the edge $t_{1} t_{2}$ will be deleted from $G^{\prime}$ by the reduction rules. Hence, the weight of vertex $v$ decreases by $\Delta_{5}$, the weight of vertex $t_{1}$ decreases by $w_{3}^{\prime}$, and the weight of vertex $t_{2}$ decreases by $\Delta_{4,3}$. Thus, the total weight decrease in the branch of force $\left(v t_{1}\right)$ is at least $w_{5}-w_{5}^{\prime}+w_{3}^{\prime}+w_{4}-w_{3}$.

In the branch of delete $\left(v t_{1}\right)$, the edge $v t_{1}$ will be deleted from $G^{\prime}$ by the branching operation, and the edge $t_{1} t_{2}$ will be added to $F^{\prime}$ by the reduction rules. Hence, the weight of vertex $v$ decreases by $\Delta_{5,4}$, the weight of vertex $t_{1}$ decreases by $w_{3}^{\prime}$, and the weight of vertex $t_{2}$ decreases by $\Delta_{4}$. Thus, the total weight decrease in the branch of delete $\left(v t_{1}\right)$ is at least $w_{5}-w_{4}+w_{3}^{\prime}+w_{4}-w_{4}^{\prime}$.

As a result, we get the following branching vector:

$$
\left(w_{5}-w_{5}^{\prime}+w_{3}^{\prime}+w_{4}-w_{3}, w_{5}+w_{3}^{\prime}-w_{4}^{\prime}\right)
$$

In the branch of force $\left(v t_{1}\right)$, the vertex $v$ will become an f5vertex, and at this point, one of the branching rules of cases c-1, c-2 and c-3 becomes immediately applicable, because an optimal edge for the next branching operation is at least as good as the edge $v t_{2}$. Therefore, for each of the 20 branching vectors $(A, B)$ of cases c-1 to c-3, we get the following branching vector:

$$
\left(w_{5}-w_{5}^{\prime}+w_{3}^{\prime}+w_{4}-w_{3}+A, w_{5}-w_{5}^{\prime}+w_{3}^{\prime}+w_{4}-w_{3}+B, w_{5}+w_{3}^{\prime}-w_{4}^{\prime}\right) .
$$

Case c-10(V). Vertex $t_{2}$ is a u5-vertex (see Fig. 20): We branch on edge $v t_{1}$.

In the branch of force $\left(v t_{1}\right)$, the edge $v t_{1}$ will be added to $F^{\prime}$ by the branching operation, and the edge $t_{1} t_{2}$ will be deleted from $G^{\prime}$ by the reduction rules. Hence, the weight of vertex $v$ decreases by $\Delta_{5}$, the weight of vertex $t_{1}$ decreases by $w_{3}^{\prime}$, and the weight of vertex $t_{2}$ decreases by $\Delta_{5,4}$. Thus, the total weight decrease in the branch of force $\left(v t_{1}\right)$ is at least $w_{5}-w_{5}^{\prime}+w_{3}^{\prime}+w_{5}-w_{4}$.

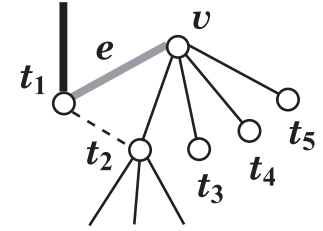

(a) force $\left(v t_{1}\right)$ in c-10(V)

: unforced edges

: forced edges

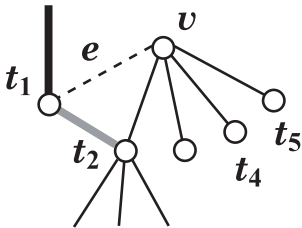

(b) delete $\left(v t_{1}\right)$ in c-10(V)

..... : newly deleted edges : newly forced edges
Fig. 20 Illustration for c-10(V) where vertices $v \in V_{\mathrm{u} 5}$ and $t_{1} \in N_{U}\left(v ; V_{\mathrm{f} 3}\right)$, such that $N_{U}(v) \cap N_{U}\left(t_{1}\right)=\left\{t_{2}\right\}, t_{2}$ is a u5-vertex.

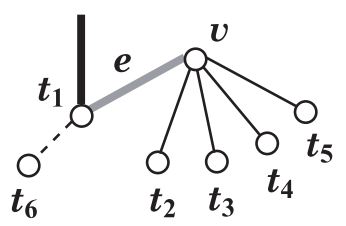

(a) force $\left(v t_{1}\right)$ in c-11

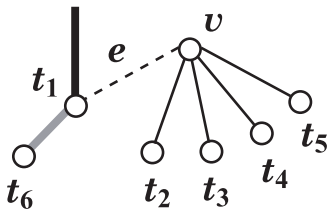

(b) delete $\left(v t_{1}\right)$ in c-11

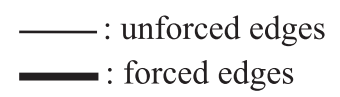

-. - . - : newly deleted edges : newly forced edges

Fig. 21 Illustration for c-11 where vertices $v \in V_{\mathrm{u} 5}$ and $t_{1} \in N_{U}\left(v ; V_{\mathrm{f} 3}\right)$.

In the branch of delete $\left(v t_{1}\right)$, the edge $v t_{1}$ will be deleted from $G^{\prime}$ by the branching operation, and the edge $t_{1} t_{2}$ will be added to $F^{\prime}$ by the reduction rules. Hence, the weight of vertex $v$ decreases by $\Delta_{5,4}$, the weight of vertex $t_{1}$ decreases by $w_{3}^{\prime}$, and the weight of vertex $t_{2}$ decreases by $\Delta_{5}$. Thus, the total weight decrease in the branch of delete $\left(v t_{1}\right)$ is at least $w_{5}-w_{4}+w_{3}^{\prime}+w_{5}-w_{5}^{\prime}$.

As a result, we get the following branching vector:

$$
\left(2 w_{5}-w_{5}^{\prime}+w_{3}^{\prime}-w_{4}, 2 w_{5}-w_{4}+w_{3}^{\prime}-w_{5}^{\prime}\right) \text {. }
$$

In the branch of force $\left(v t_{1}\right)$, the vertex $v$ will become an f5vertex, and at this point, one of the branching rules of cases c-1 to c- 6 becomes immediately applicable, because an optimal edge for the next branching operation is at least as good as the edge $v t_{2}$. Therefore, for each of the 24 branching vectors $(A, B)$ of cases c- 1 to $\mathrm{c}-6$, we get the following branching vector:

$$
\left(2 w_{5}-w_{5}^{\prime}+w_{3}^{\prime}-w_{4}+A, 2 w_{5}-w_{5}^{\prime}+w_{3}^{\prime}-w_{4}+B, 2 w_{5}-w_{4}+w_{3}^{\prime}-w_{5}^{\prime}\right) .
$$

Case c-11. None of the previous cases are applicable, and there exist vertices $v \in V_{\mathrm{u} 5}$ and $t_{1} \in N_{U}\left(v ; V_{\mathrm{f} 3}\right)$ such that $N_{U}(v) \cap N_{U}\left(t_{1}\right)=\emptyset$ (see Fig. 21): We branch on edge $v t_{1}$. Note that $N_{U}\left(t_{1}\right) \backslash\{v\}=\left\{t_{6}\right\}$.

In the branch of force $\left(v t_{1}\right)$, the edge $v t_{1}$ will be added to $F^{\prime}$ by the branching operation, and the edge $t_{1} t_{6}$ will be deleted from $G^{\prime}$ by the reduction rules. So, the weight of vertex $v$ decreases by $\Delta_{5}$, and the weight of vertex $t_{1}$ decreases by $w_{3}^{\prime}$.

In the branch of delete $\left(v t_{1}\right)$, the edge $v t_{1}$ will be deleted from $G^{\prime}$ by the branching operation, and the edge $t_{1} t_{6}$ will be added to $F^{\prime}$ by the reduction rules. The weight of vertex $v$ decreases by $\Delta_{5,4}$, and the weight of vertex $t_{1}$ decreases by $w_{3}^{\prime}$.

There are two cases for the vertex $t_{6}$; a) vertex $t_{6}$ is of type $\mathrm{f} 3$, and b) otherwise. We analyze the branches force $\left(v t_{1}\right)$ and $\operatorname{delete}\left(v t_{1}\right)$ for these two cases separately.

First, we analyze the case where the vertex $t_{6}$ is an f3-vertex 
(see Fig. 2). Recall that in such a case we denote by $x$ the unique neighbor of $t_{6}$ different from $t_{1}$. In the branch of force $\left(v t_{1}\right)$, the edge $x t_{6}$ will be added to $F^{\prime}$ by the reduction rules. Hence, the weight of vertex $t_{6}$ decreases by $w_{3}^{\prime}$. If the vertex $x$ is an $\mathrm{f} 3$-vertex (resp., u3, f4, u4, and a u5-vertex), then the weight decrease $\alpha_{5}$ of vertex $x$ will be $w_{3}^{\prime}$ (resp., $\Delta_{3}, w_{4}^{\prime}, \Delta_{4}$, and $\Delta_{5}$ ). Thus, the total weight decrease for this case in the branch of force $\left(v t_{1}\right)$ is at least $w_{5}-w_{5}^{\prime}+w_{3}^{\prime}+w_{3}^{\prime}+\alpha_{5}$.

In the branch of delete $\left(v t_{1}\right)$, the edge $x t_{6}$ will be deleted from $G^{\prime}$ by the reduction rules. Hence, the weight of vertex $t_{6}$ decreases by $w_{3}^{\prime}$. If the vertex $x$ is an f3-vertex (resp., u3, f4, u4, and a u5-vertex), then the weight decrease $\beta_{5}$ of vertex $x$ will be $w_{3}^{\prime}$ (resp., $w_{3}, \Delta_{4,3}^{\prime}, \Delta_{4,3}$, and $\Delta_{5,4}$ ). Thus, the total weight decrease for the case where $t_{6}$ is an f3-vertex in the branch of delete $\left(v t_{1}\right)$ is at least $w_{5}-w_{4}+w_{3}^{\prime}+w_{3}^{\prime}+\beta_{5}$.

As a result, for the ordered pair $\left(\alpha_{5}, \beta_{5}\right)$ taking values in $\left\{\left(w_{3}^{\prime}, w_{3}^{\prime}\right),\left(\Delta_{3}, w_{3}\right),\left(w_{4}^{\prime}, \Delta_{4,3}^{\prime}\right),\left(\Delta_{4}, \Delta_{4,3}\right),\left(\Delta_{5}, \Delta_{5,4}\right)\right\}$, we get the following five branching vectors:

$$
\left(w_{5}-w_{5}^{\prime}+2 w_{3}^{\prime}+\alpha_{5}, w_{5}-w_{4}+2 w_{3}^{\prime}+\beta_{5}\right)
$$

In the branch of force $\left(v t_{1}\right)$, the vertex $v$ will become an f5vertex, and at this point, one of the branching rules of cases c-1 to c-9 becomes immediately applicable. Therefore, for the case where the vertex $t_{6}$ is an $\mathrm{f} 3$-vertex, for each of the 29 branching vectors $(A, B)$ of cases $\mathrm{c}-1$ to $\mathrm{c}-9$, we get the following branching vector:

$$
\left(w_{5}-w_{5}^{\prime}+2 w_{3}^{\prime}+\alpha_{5}+A, w_{5}-w_{5}^{\prime}+2 w_{3}^{\prime}+\alpha_{5}+B, w_{5}-w_{4}+2 w_{3}^{\prime}+\beta_{5}\right) .
$$

Next, we examine the case where the vertex $t_{6}$ is not an f3vertex. In the branch of force $\left(v t_{1}\right)$, if the vertex $t_{6}$ is a u3-vertex (resp., f4, u4, and a u5-vertex), then the weight decrease $\alpha_{6}$ of vertex $t_{6}$ will be $w_{3}$ (resp., $\Delta_{4,3}^{\prime}, \Delta_{4,3}$, and $\Delta_{5,4}$ ). Thus, the total weight decrease for this case in the branch of force $\left(v t_{1}\right)$ is at least $w_{5}-w_{5}^{\prime}+w_{3}^{\prime}+\alpha_{6}$.

In the branch of delete $\left(v t_{1}\right)$, if the vertex $t_{6}$ is a u3-vertex (resp., $\mathrm{f} 4, \mathrm{u} 4$, and a u5-vertex), then the weight decrease $\beta_{6}$ of vertex $t_{6}$ will be $\Delta_{3}$ (resp., $w_{4}^{\prime}, \Delta_{4}$, and $\Delta_{5}$ ). Thus, the total weight decrease for the case where $t_{6}$ is not an f3-vertex in the branch of $\operatorname{delete}\left(v t_{1}\right)$ is at least $w_{5}-w_{4}+w_{3}^{\prime}+\beta_{6}$.

As a result, for the ordered pair $\left(\alpha_{6}, \beta_{6}\right)$ taking values in $\left\{\left(w_{3}, \Delta_{3}\right),\left(\Delta_{4,3}^{\prime}, w_{4}^{\prime}\right),\left(\Delta_{4,3}, \Delta_{4}\right),\left(\Delta_{5,4}, \Delta_{5}\right)\right\}$, we get the following four branching vectors:

$$
\left(w_{5}-w_{5}^{\prime}+w_{3}^{\prime}+\alpha_{6}, w_{5}-w_{4}+w_{3}^{\prime}+\beta_{6}\right) .
$$

In the branch of force $\left(v t_{1}\right)$, for each of the 29 branching vectors $(A, B)$ of cases c-1 to c-9, we get the following branching vector:

$$
\left(w_{5}-w_{5}^{\prime}+w_{3}^{\prime}+\alpha_{6}+A, w_{5}-w_{5}^{\prime}+w_{3}^{\prime}+\alpha_{6}+B, w_{5}-w_{4}+w_{3}^{\prime}+\beta_{6}\right) .
$$

Case c-12. None of the previous cases are applicable, and there exist vertices $v \in V_{\mathrm{u} 5}$ and $t \in N_{U}\left(v ; V_{\mathrm{u} 3}\right)$ (see Fig. 22): We branch on edge $v t_{1}$. Note that $N_{U}\left(t_{1}\right) \backslash\{v\}=\left\{t_{6}, t_{7}\right\}$.

In the branch of force $\left(v t_{1}\right)$, the edge $v t_{1}$ will be added to $F^{\prime}$ by the branching operation. So, the weight of vertex $v$ decreases by

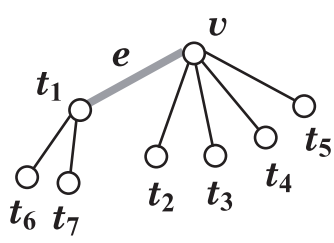

(a) force $\left(v t_{1}\right)$ in c-12

: unforced edges

: forced edges

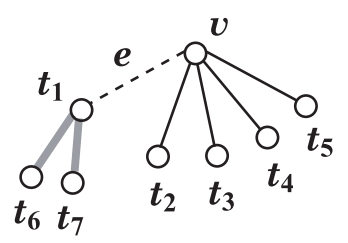

(b) delete $\left(v t_{1}\right)$ in c-12 : newly deleted edges : newly forced edges
Fig. 22 Illustration of branching rule c-12, where vertex $v \in V_{\mathrm{u} 5}$ and vertex $t \in N_{U}\left(v ; V_{\mathrm{u} 3}\right)$.

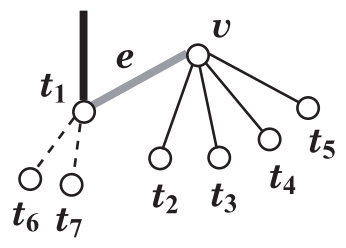

(a) force $\left(v t_{1}\right)$ in c-13

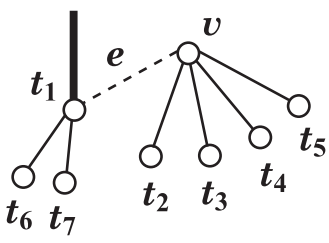

(b) delete $\left(v t_{1}\right)$ in c-13

\section{_ : unforced edges $\quad . . . . .$. : newly deleted edges \\ : forced edges \\ : newly forced edges \\ Fig. 23 Illustration for c-13 where vertices $v \in V_{\mathrm{u} 5}$ and $t_{1} \in N_{U}\left(v ; V_{\mathrm{f} 4}\right)$.}

$\Delta_{5}$, and the weight of vertex $t_{1}$ decreases by $\Delta_{3}$. The total weight decrease in the branch of force $\left(v t_{1}\right)$ is at least $w_{5}-w_{5}^{\prime}+w_{3}-w_{3}^{\prime}$.

In the branch of delete $\left(v t_{1}\right)$, the edge $v t_{1}$ will be deleted from $G^{\prime}$ by the branching operation, and edges $t_{1} t_{6}$ and $t_{1} t_{7}$ will be added to $F^{\prime}$ by the reduction rules. So, the weight of vertex $v$ decreases by $\Delta_{5,4}$, and the weight of vertex $t_{1}$ decreases by $w_{3}$. Each of the vertices $t_{6}$ and $t_{7}$ must be one of types f3, u3, f4, u4, and a u5-vertex, and each of their weights decreases by at least $m_{9}=\min \left\{w_{3}^{\prime}, \Delta_{3}, w_{4}^{\prime}, \Delta_{4}, \Delta_{5}\right\}$. Thus, the total weight decrease in the branch of $\operatorname{delete}\left(v t_{1}\right)$ is at least $w_{5}-w_{4}+w_{3}+2 m_{9}$.

As a result, we get the following branching vector:

$$
\left(w_{5}-w_{5}^{\prime}+w_{3}-w_{3}^{\prime}, w_{5}-w_{4}+w_{3}+2 m_{9}\right) .
$$

In the branch of force $\left(v t_{1}\right)$, the vertex $v$ will become an f5vertex, and at this point, one of the branching rules of cases c-3 to c- 6 and c- 9 becomes immediately applicable, since there are no more $\mathrm{f} 3$ vertices and no other f5 vertices, thus cases c-1, c-2, c-7 and c- 8 cannot occur following this branching operation. Therefore, for each of the six branching vectors $(A, B)$ of cases c-3 to c- 6 and case c- 9 , we get the following branching vector:

$$
\left(w_{5}-w_{5}^{\prime}+w_{3}-w_{3}^{\prime}+A, w_{5}-w_{5}^{\prime}+w_{3}-w_{3}^{\prime}+B, w_{5}-w_{4}+w_{3}+2 m_{9}\right) .
$$

Case c-13. None of the previous cases are applicable, and there exist vertices $v \in V_{\mathrm{u} 5}$ and $t_{1} \in N_{U}\left(v ; V_{\mathrm{f} 4}\right)$ (see Fig. 23): We branch on edge $v t_{1}$. Note that $N_{U}\left(t_{1}\right) \backslash\{v\}=\left\{t_{6}, t_{7}\right\}$.

In the branch of force $\left(v t_{1}\right)$, the edge $v t_{1}$ will be added to $F^{\prime}$ by the branching operation, and edges $t_{1} t_{6}$ and $t_{1} t_{7}$ will be deleted from $G^{\prime}$ by the reduction rules. So, the weight of vertex $v$ decreases by $\Delta_{5}$, and the weight of vertex $t_{1}$ decreases by $w_{4}^{\prime}$. Each of the vertices $t_{6}$ and $t_{7}$ must be one of types $\mathrm{f} 3, \mathrm{u} 3, \mathrm{f} 4, \mathrm{u} 4$, and a u5-vertex, and each of their weights decreases by at least $m_{10}=\min \left\{w_{3}^{\prime}, w_{3}, \Delta_{4,3}^{\prime}, \Delta_{4,3}, \Delta_{5,4}\right\}$. Thus, the total weight decrease in the branch of force $\left(v t_{1}\right)$ is at least $w_{5}-w_{5}^{\prime}+w_{4}^{\prime}+2 m_{10}$. 


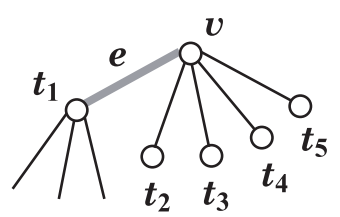

(a) force $\left(v t_{1}\right)$ in c-14 : unforced edges forced edges

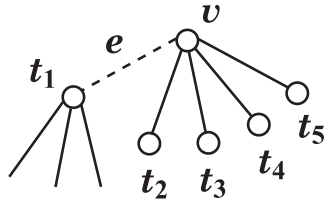

(b) delete $\left(v t_{1}\right)$ in c-14

..... : newly deleted edges : newly forced edges
Fig. 24 Illustration of branching rule c-14, where vertex $v \in V_{\mathrm{u} 5}$ and vertex $t_{1} \in N_{U}\left(v ; V_{\mathrm{u} 4}\right)$

In the branch of delete $\left(v t_{1}\right)$, the edge $v t_{1}$ will be deleted from $G^{\prime}$ by the branching operation. So, the weight of vertex $v$ decreases by $\Delta_{5,4}$, and the weight of vertex $t_{1}$ decreases by $\Delta_{4,3}^{\prime}$. The total weight decrease in the branch of $\operatorname{delete}\left(v t_{1}\right)$ is at least $w_{5}-w_{4}+w_{4}^{\prime}-w_{3}^{\prime}$.

As a result, we get the following branching vector:

$$
\left(w_{5}-w_{5}^{\prime}+w_{4}^{\prime}+2 m_{10}, w_{5}-w_{4}+w_{4}^{\prime}-w_{3}^{\prime}\right) .
$$

In the branch of force $\left(v t_{1}\right)$, the vertex $v$ will become an f5vertex, and at this point, one of the branching rules of cases c-4 to c-9 becomes immediately applicable. Therefore, for each of the nine branching vectors $(A, B)$ of cases c-4 to c-9, we get the following branching vector:

$$
\left(w_{5}-w_{5}^{\prime}+w_{4}^{\prime}+2 m_{10}+A, w_{5}-w_{5}^{\prime}+w_{4}^{\prime}+2 m_{10}+B, w_{5}-w_{4}+w_{4}^{\prime}-w_{3}^{\prime}\right) .
$$

Case c-14. None of the previous cases are applicable, and there exist vertices $v \in V_{\mathrm{u} 5}$ and $t_{1} \in N_{U}\left(v ; V_{\mathrm{u} 4}\right)$ (see Fig. 24): We branch on edge $v t_{1}$.

In the branch of force $\left(v t_{1}\right)$, the edge $v t_{1}$ will be added to $F^{\prime}$ by the branching operation. So, the weight of vertex $v$ decreases by $\Delta_{5}$, and the weight of vertex $t_{1}$ decreases by $\Delta_{4}$. Thus, the total weight decrease in the branch of force $\left(v t_{1}\right)$ is at least $w_{5}-w_{5}^{\prime}+w_{4}-w_{4}^{\prime}$.

In the branch of delete $\left(v t_{1}\right)$, the edge $v t_{1}$ will be deleted from $G^{\prime}$ by the branching operation. So, the weight of vertex $v$ decreases by $\Delta_{5,4}$, and the weight of vertex $t_{1}$ decreases by $\Delta_{4,3}$. Thus, the total weight decrease in the branch of $\operatorname{delete}\left(v t_{1}\right)$ is at least $w_{5}-w_{3}$.

Then, we get the following branching vector:

$$
\left(w_{5}-w_{5}^{\prime}+w_{4}-w_{4}^{\prime}, w_{5}-w_{3}\right)
$$

In the branch of force $\left(v t_{1}\right)$, the vertex $v$ will become an f5vertex, and at this point, one of the branching rules of cases c- 6 and c- 9 becomes immediately applicable, since there are only $\mathrm{u} 4$ and $\mathrm{u} 5$ vertices that remain, and thus only cases c- 6 and c- 9 can occur following to this branching operation. Therefore, for each of the two branching vectors $(A, B)$ of cases c-6 and c-9, we get the following branching vector:

$$
\left(w_{5}-w_{5}^{\prime}+w_{4}-w_{4}^{\prime}+A, w_{5}-w_{5}^{\prime}+w_{4}-w_{4}^{\prime}+B, w_{5}-w_{3}\right) .
$$

Case c-15. None of the previous cases are applicable, and there exist vertices $v \in V_{\mathrm{u} 5}$ and $t_{1} \in N_{U}\left(v ; V_{\mathrm{u} 5}\right)$ (see Fig. 25): We branch

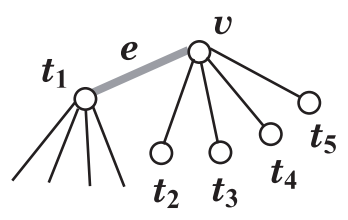

(a) force $\left(v t_{1}\right)$ in c-15

: unforced edges forced edges

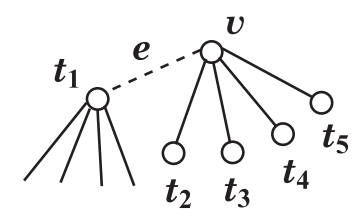

(b) delete $\left(v t_{1}\right)$ in c-15

-...-: newly deleted edges : newly forced edges
Fig. 25 Illustration of branching rule c-15, where vertex $v \in V_{\mathrm{u} 5}$ and vertex $t_{1} \in N_{U}\left(v ; V_{\mathrm{u} 5}\right)$.

on edge $v t_{1}$.

In the branch of force $\left(v t_{1}\right)$, the edge $v t_{1}$ will be added to $F^{\prime}$ by the branching operation. So, both weights of vertex $v$ and vertex $t_{1}$ each decrease by $\Delta_{5}$. Thus, the total weight decrease in the branch of force $\left(v t_{1}\right)$ is at least $2 w_{5}-2 w_{5}^{\prime}$.

In the branch of delete $\left(v t_{1}\right)$, the edge $v t_{1}$ will be deleted from $G^{\prime}$ by the branching operation. So, both weights of vertex $v$ and vertex $t_{1}$ each decrease by $\Delta_{5,4}$. Thus, the total weight decrease in the branch of $\operatorname{delete}\left(v t_{1}\right)$ is at least $2 w_{5}-2 w_{4}$.

Then, we get the following branching vector:

$$
\left(2 w_{5}-2 w_{5}^{\prime}, 2 w_{5}-2 w_{4}\right) \text {. }
$$

In the branch of force $\left(v t_{1}\right)$, the vertex $v$ will become an 55 vertex, and at this point, the branching rule of case c- 9 becomes immediately applicable, since there are only u5 vertices that remain, and thus only case c- 9 can occur following to this branching operation. Therefore, for the branching vector $(A, B)$ of case $\mathrm{c}-9$, we get the following branching vector:

$\left(2 w_{5}-2 w_{5}^{\prime}+A, 2 w_{5}-2 w_{5}^{\prime}+B, 2 w_{5}-2 w_{4}\right)$.

\subsection{Switching to TSP in Degree-4 Graphs}

If none of the above 15 cases can be executed, then this means that the graph has no more degree- 5 vertices and the maximum degree of the graph at this point is 4 or less. In that case, we can switch and use a fast algorithm for TSP in degree- 4 graphs, called tsp4 $(G, F)$ to solve the remaining instance $I=(G, F)$. Since at the point when $\operatorname{tsp} 4(G, F)$ is called the instance $I=(G, F)$ is a reduced instance, it has no vertices of degree 1 or less, nor vertices with three or more incident forced edges. Moreover, without loss of generality we can assume that there is also no vertex $v \in V(G)$ of degree 2, as its weight $\omega(v)$ is set to be 0 in Eq. (18), and together with the two forced edges incident with $v$ can be replaced by a single forced edge [15], [16]. Then, let $n_{3}^{\prime}, n_{3}, n_{4}^{\prime}$ and $n_{4}$ be the numbers of $\mathrm{f} 3, \mathrm{u} 3, \mathrm{f} 4$ and $\mathrm{u} 4$ vertices in $I$, respectively, where $n_{3}^{\prime}+n_{3}+n_{4}^{\prime}+n_{4}=|V(G)|$, and by Eq. (1) the measure $\mu(I)$ of instance $I$ is $\mu(I)=w_{3}^{\prime} n_{3}^{\prime}+w_{3} n_{3}+w_{4}^{\prime} n_{4}^{\prime}+w_{4} n_{4}$.

At this step, we switch to use the $O^{*}\left(1.69193^{n}\right)$-time algorithm for the TSP in degree-4 graphs by Xiao and Nagamochi [16]. Let $\hat{\mu}(I)$ denote the measure of the TSP in the degree- 4 instance $I$, where $\hat{\mu}(I)=\hat{w}_{3}^{\prime} n_{3}^{\prime}+\hat{w}_{3} n_{3}+\hat{w}_{4}^{\prime} n_{4}^{\prime}+\hat{w}_{4} n_{4}$, and the weight $\hat{w}_{3}^{\prime}$ for an $\mathrm{f} 3$-vertex is 0.21968 , the weight $\hat{w}_{3}$ for an u3-vertex is 0.45540 , the weight $\hat{w}_{4}^{\prime}$ for an f4-vertex is 0.59804 and the weight $\hat{w}_{4}$ for an $\mathrm{u} 4$-vertex is 1 [16]. Hence, the running time bound of the TSP in the degree-4 instance $I$ with measure $\hat{\mu}(I)$ is given by $1.69193^{\hat{\mu}(I)}$. Since the bound on the running time of $\operatorname{tsp} 5(G, F)$ is expressed 
by $\tau^{\mu(I)}$, we get a constraint on the value of $\tau$ :

$$
1.69193^{\hat{\mu}(I)} \leq \tau^{\mu(I)}
$$

for any partition $\left\{n_{3}^{\prime}, n_{3}, n_{4}^{\prime}, n_{4}\right\}$ of $|V(G)|$. In other words,

$$
\begin{aligned}
& 1.69193^{0.21968} \leq \tau^{w_{3}^{\prime}}, \\
& 1.69193^{0.45540} \leq \tau^{w_{3}}, \\
& 1.69193^{0.59804} \leq \tau^{w_{4}^{\prime}}, \text { and } \\
& 1.69193^{1} \leq \tau^{w_{4}} .
\end{aligned}
$$

Equations (45) to (48) will become constraints in the quasiconvex program.

\subsection{Overall Analysis}

The branching factors of each of the branching vectors from Eqs. (19) to (44) and the switching constraints of Eqs. (45) to (48) do not exceed 2.349978. The tight constraints in the quasiconvex program are in conditions $\mathrm{c}-11, \mathrm{c}-12, \mathrm{c}-14, \mathrm{c}-15$ and the switching constraints. This completes the proof of Lemma 2, and therefore, Theorem 1.

\section{Conclusion}

In this paper, we have presented an exact algorithm for TSP in degree-5 graphs. Our algorithm is a simple branching algorithm, following the branch-and-reduce paradigm, and it can be implemented to operate in space which is polynomial of the size of an input instance. For the analysis of the running time of the proposed algorithm, we used the measure and conquer method, and have obtained an upper bound of $O^{*}\left(2.3500^{n}\right)$, where $n$ is the number of vertices in a given instance. This result improves a previous time bound on the TSP in degree- 5 graphs by Md Yunos et al. [13].

The greatest challenge in obtaining a non-trivial time-bound for the algorithm is to derive a proper case analysis, and the interdependence of the branching rules makes the problem ever more difficult with graphs of higher degree. In contrast, the above results offer a purely theoretically improved time-bound on the running time. With regards to a recent result by Akiba and Iwata [1], algorithms with theoretically improved running time can indeed be also superior in practice, and it would be of much interest to implement the proposed algorithms and evaluate their empirical performance. This branch of research would also open the question of devising bounding rules which might not have an impact on the theoretical running time bound, but might contribute meaningfully to the algorithm's performance in practice.

\section{References}

[1] Akiba, T. and Iwata, Y.: Branch-and-reduce Exponential/FPT Algorithms in Practice: A Case Study of Vertex Cover, Proc. 17th Workshop on Algorithm Engineering and Experiments (ALENEX), SIAM, pp.70-81 (2015).

[2] Bellman, R.E.: Combinatorial Processes and Dynamic Programming, Combinatorial Analysis, Proc. Symposia in Applied Mathematics 10, American Mathematical Society, pp.217-249 (1960).

[3] Bodlaender, H.L., Cygan, M., Kratsch, S. and Nederlof, J.: Deterministic Single Exponential Time Algorithms for Connectivity Problems Parameterized by Treewidth, Information and Computation, Vol.243, pp.86-111 (2015).

[4] Eppstein, D.: Quasiconvex Analysis of Backtracking Algorithms,
Proc. 15th Annual ACM-SIAM Symposium On Discrete Algorithms (SODA 2004), pp.781-790, ACM Press (2004).

[5] Eppstein, D.: The Traveling Salesman Problem for Cubic Graphs, Journal of Graph Algorithms and Application, Vol.11, No.1, pp.6181 (2007).

[6] Fomin, F.V., Grandoni, F. and Kratsch, D.: A Measure and Conquer Approach for the Analysis of Exact Algorithms, J. ACM, Vol.56, No.5, Article No.25 (2009).

[7] Fomin, F.V. and Kratsch, D.: Exact Exponential Algorithms, Springer (2010).

[8] Gebauer, H.: Finding and Enumerating Hamilton Cycles in 4-regular Graphs, Theoretical Computer Science, Vol.412, No.35, pp.45794591 (2011).

[9] Gurevich, Y. and Shelah, S.: Expected Computation Time for Hamiltonian Path Problem, SIAM Journal of Computation, Vol.16, No.3, pp.486-502 (1987).

[10] Held, M. and Karp, R.M.: A Dynamic Programming Approach to Sequencing Problems, Journal of the Society for Industrial and Applied Mathematics, Vol.10, No.1, pp.196-210 (1962).

[11] Iwama, K. and Nakashima, T.: An Improved Exact Algorithm for Cubic Graph TSP, Computing and Combinatorics, Lecture Notes in Computer Science, Vol.4598, pp.108-117 (2007).

[12] Liskiewicz, M. and Schuster, M.R.: A New Upper Bound for the Traveling Salesman Problem in Cubic Graphs, Journal of Discrete Algorithms, Vol.27, pp.1-20 (2014).

[13] Md Yunos, N., Shurbevski, A. and Nagamochi, H.: A Polynomialspace Exact Algorithm for TSP in Degree-5 Graphs, The 12th International Symposium on Operations Research and Its Application (ISORA 2015), pp.45-58 (2015).

[14] Rubin, F.: A Search Procedure for Hamilton Paths and Circuits, J. ACM, Vol.21, No.4, pp.576-580 (1974).

[15] Xiao, M. and Nagamochi, H.: An Exact Algorithm for TSP in Degree3 Graphs via Circuit Procedure and Amortization on Connectivity Structure, Algorithmica, Vol.74, No.2, pp.713-741 (2016).

[16] Xiao, M. and Nagamochi, H.: An Improved Exact Algorithm for TSP in Graphs of Maximum Degree 4, Theory of Computing Systems, Vol.58, No.2, pp.241-272 (2016).

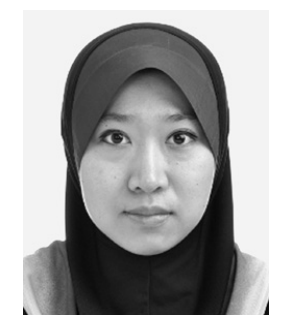

Norhazwani Md Yunos was born in 1985. She received her M.Sc. degree from the University of Technology, Malaysia in 2009, and a Ph.D. degree in Informatics from Kyoto University in 2017. Currently, she works at the Technical University of Malaysia Malacca, Malacca, Malaysia as a lecturer, resuming her position from before she left Malaysia to pursue her Ph.D. studies in Japan. Her research interests include Combinatorial Optimization, Routing and Scheduling, etc.

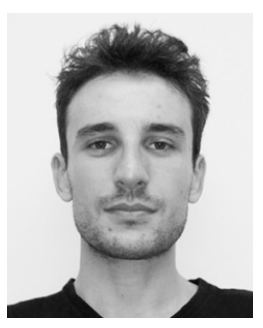

Aleksandar Shurbevski was born in 1986. He received his M.Sc. degree from Kyoto Institute of Technology in 2012, and a Ph.D. degree in Informatics from Kyoto University in 2014. As of 2014 he is an Assistant Professor at the Department of Applied Mathematics and Physics, Graduate School of Informatics, Kyoto University, and his research interests include Combinatorial Optimization, Graph Algorithms, etc. He is a member of IEEE and JSOR. 


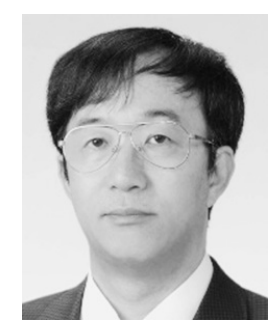

Hiroshi Nagamochi was born in 1960. He received his D.E. degree from Kyoto University in 1988. He became a Professor in the Department of Applied Mathematics and Physics, Graduate School of Informatics, Kyoto University in 2004 after serving for Toyohashi University of Technology. His research interests include Discrete Optimization, Graph Algorithms, Scheduling, Packing Problems, Chemical Graphs, etc. 